2013

\title{
On Lagrangian Solutions for the Semi-geostrophic System with Singular Initial Data
}

Mikhail Feldman

Adrian Tudorascu

Follow this and additional works at: https://researchrepository.wvu.edu/faculty_publications

\section{Digital Commons Citation}

Feldman, Mikhail and Tudorascu, Adrian, "On Lagrangian Solutions for the Semi-geostrophic System with Singular Initial Data" (2013). Faculty Scholarship. 258.

https://researchrepository.wvu.edu/faculty_publications/258

This Article is brought to you for free and open access by The Research Repository @ WVU. It has been accepted for inclusion in Faculty Scholarship by an authorized administrator of The Research Repository@ WVU. For more information, please contact ian.harmon@mail.wvu.edu. 


\title{
On Lagrangian solutions for the semi-geostrophic system with singular initial data *
}

\author{
Mikhail Feldman \\ Department of Mathematics \\ University of Wisconsin-Madison \\ Madison, WI 53706, USA \\ feldman@math.wisc.edu \\ Adrian Tudorascu \\ Department of Mathematics \\ West Virginia University \\ Morgantown, WV 26506, USA \\ adriant@math.wvu.edu
}

January 8, 2013

\begin{abstract}
We show that weak (Eulerian) solutions for the Semi-Geostrophic system in physical space exhibiting some mild regularity in time cannot yield point masses in dual space. However, such solutions are physically relevant to the model. Thus, we discuss a natural generalization of weak Lagrangian solutions in the physical space to include the possibility of singular measures in dual space. We prove existence of such solutions in the case of discrete measures in dual space. We also prove that weak Lagrangian solutions in physical space determine solutions in the dual space. This implies conservation of geostrophic energy along the Lagrangian trajectories in the physical space.
\end{abstract}

\section{Introduction}

The Semi-Geostrophic (abbreviated SG in this work) equations have been proposed as simplifications of the primitive equations (Boussinesq) when the rate of change of momentum is much smaller than the Coriolis "force" (small Rossby number) [16]. The advected quantity momentum is approximated by its geostrophic value, but the trajectories are not.

Throughout the entire paper, $\Omega \subset \mathbb{R}^{3}$ is a given open, bounded set, and $T \in(0, \infty)$ is fixed. A

${ }^{*}$ Key words: SG system, flows of maps, optimal mass transport, Wasserstein metric, optimal maps, absolutely continuous curves. 
version of the 3D Semi-Geostrophic system $[6,9]$ is

$$
\begin{aligned}
& D_{t} X=J[X-x], \\
& X=\nabla P, \\
& \nabla \cdot u=0, \\
& u \cdot \nu=0, \quad \text { on }[0, T) \times \partial \Omega, \\
& P(0, \cdot)=P_{0} \quad \text { in } \Omega,
\end{aligned}
$$

where $P_{0}$ is convex defined on $\Omega$. One looks for solutions $(P, u)$ satisfying the Cullen-Purser stability condition (see, e.g., [10]), which amounts to imposing that $P_{t}(\cdot):=P(t, \cdot)$ be convex for all $t \in[0, T)$. Here,

$$
J=\left(\begin{array}{rrr}
0 & -1 & 0 \\
1 & 0 & 0 \\
0 & 0 & 0
\end{array}\right) .
$$

Henceforth, we shall assume without loss of generality that $\mathcal{L}^{3}(\Omega)=1$ (otherwise all the measures considered will have total mass equal to the volume of $\Omega$ ) and denote

$$
\chi:=\left.\mathcal{L}^{3}\right|_{\Omega} .
$$

If $\nabla P_{t \#} \chi=: \alpha_{t}$ are all absolutely continuous with respect to $\mathcal{L}^{3}$, then one can use the LegendreFenchel transforms $P_{t}^{*}$ of $P_{t}$ to formally rewrite (1) as the so-called SG in dual variables

$$
\begin{aligned}
& \partial_{t} \alpha+\nabla \cdot(U \alpha)=0 \quad \text { in }[0, T) \times \mathbb{R}^{3}, \\
& \nabla P(t, \cdot) \# \chi=\alpha(t, \cdot) \quad \text { for any } t \in[0, T) \\
& U(t, X)=J\left[X-\nabla P^{*}(t, X)\right] \\
& \alpha(0, X)=\alpha_{0}(X) \quad \text { for a.e. } X \in \mathbb{R}^{3} .
\end{aligned}
$$

Existence of solutions of the problem recast in dual variables was obtained by J. D. Benamou, Y. Brenier [6], and for some related models by M. Cullen and W. Gangbo [10], and M. Cullen and H. Maroofi [12]. They considered case when $\alpha_{0} \in L^{q}(\Omega)$ for some $q>1$, and the solution satisfies $\alpha(t, \cdot) \in L^{q}$ for all $t$. These results were extended to the case $q=1$ in [18].

These solutions are not known to be regular enough to be translated into Eulerian solutions of the problem in physical space. Recently, existence of Eulerian solutions for a class of initial data, where the conditions include the requirement that the support of $\alpha_{0}=\nabla P_{0 \#} \chi$ in the dual space is the whole space, was obtained by L. Ambrosio, M. Colombo, G. De Philippis, A. Figalli $[2,3]$ based on the results of G. De Philippis and M. Figalli [13] on regularity of solutions for the Monge-Ampere equation. Existence of Eulerian solutions in physical space for more general initial data, when the support of $\alpha_{0}$ in the dual space may have a non-empty boundary, is presently not known.

Another approach is to consider Lagrangian solutions in the physical space. Such solutions were introduced by M. Cullen and M. Feldman [9], and existence of Lagrangian solutions of (1) was shown in [9] for the case $\alpha_{0}=\nabla P_{0 \#} \chi \in L^{q}(\Omega)$ for $q>1$, on the basis of Ambrosio's theory on transport equations and flows of $B V$ vector fields [1]. These results were extended to the case $q=1$ in [14]. The definition of weak Lagrangian solutions in the physical space is following (where we slightly modify the definition given in [9] by relaxing assumptions on $P$ ).

Definition 1.1. Let $P_{0} \in H^{1}(\Omega)$ be convex, $F:[0, T) \times \Omega \rightarrow \Omega$ be a Borel map such that $F \in C\left([0, T) ; L^{2}\left(\Omega ; \mathbb{R}^{3}\right)\right)$, and let $P \in C\left([0, T) ; H^{1}(\Omega)\right)$. Assume $P(t, \cdot)$ is convex in $\Omega$ for each $t \in[0, T)$. Then the pair $(P, F)$ is called a weak Lagrangian solution of (1) in $[0, T) \times \Omega$ if 
i. $F(0, x)=x, P(0, x)=P_{0}(x)$ for a.e. $x \in \Omega$,

ii. for any $t>0$ the mapping $F_{t}=F(t, \cdot): \Omega \rightarrow \Omega$ is Lebesgue measure preserving, in the sense that $F_{t \#} \chi=\chi$;

iii. There exists a Borel map $F^{*}:[0, T) \times \Omega \rightarrow \Omega$ such that for every $t \in(0, T)$ the map $F_{t}^{*}=$ $F^{*}(t, \cdot): \Omega \rightarrow \Omega$ is Lebesgue measure preserving: $F_{t \#}^{*} \chi=\chi$, and satisfies $F_{t}^{*} \circ F_{t}(x)=x$ and $F_{t} \circ F_{t}^{*}(x)=x$ for a.e. $x \in \Omega$;

iv. The function

$$
Z(t, x)=\nabla P\left(t, F_{t}(x)\right)
$$

is a distributional solution of

$$
\begin{array}{ll}
\partial_{t} Z(t, x)=J[Z(t, x)-F(t, x)] & \text { in }[0, T) \times \Omega, \\
Z(0, x)=\nabla P_{0}(x) & \text { in } \Omega .
\end{array}
$$

Note that the sense in which (7) must be satisfied is

$$
\int_{0}^{T} \int_{\Omega}\left[Z(t, x) \cdot \partial_{t} \varphi(t, x)+J[Z(t, x)-F(t, x)] \cdot \varphi(t, x)\right] d x d t+\int_{\Omega} \nabla P_{0}(x) \cdot \varphi(0, x) d x=0
$$

for any $\varphi \in C_{c}^{1}\left([0, T) \times \Omega ; \mathbb{R}^{3}\right)$.

In this paper we consider the case of singular initial data, i.e. when $\alpha_{0}=\nabla P_{0_{\#}} \chi$ is a singular measure. The dual problem in this case was studied by G. Loeper [17], and L. Ambrosio and W. Gangbo [4].

Note that equation (2) represents the fact that the dual flow $t \rightarrow \alpha_{t}$ is weakly (in the sense of distributions) transported by the dual velocity $U$ defined by (4). The change of variable $X=\nabla P_{t}(x)$ is reversible (although there may not be enough smoothness to transport the dual space solutions back to physical space even in this case) if and only if $\nabla P_{t}^{*}$ pushes $\alpha_{t}$ forward to $\chi$, one such situation being provided if $\alpha_{t} \ll \mathcal{L}^{3}$. In general, if $\nabla P_{t}^{*}$ is not necessarily the a.e. inverse of $\nabla P_{t}$, then $\nabla P_{t}^{*}$ may not necessarily push $\alpha_{t}$ forward to $\chi, \alpha_{t}$ may not necessarily be absolutely continuous with respect to $\mathcal{L}^{3}$ and the equation (4) in the dual-variable system must be generalized to (see [4])

$$
U(t, X)=J\left[X-\bar{\gamma}_{t}(X)\right]
$$

where $\bar{\gamma}_{t}$ is the barycentric projection onto $\alpha_{t}$ of the (unique) optimal Kantorovich plan [20] $\gamma_{t}:=\left(\nabla P_{t} \times \mathrm{Id}\right)_{\#} \chi$ having $\alpha_{t}$ and $\chi$ as first and second marginals, respectively. It is defined by (see [4], [5])

$$
\int_{\mathbb{R}^{3}} \xi(X) \cdot \bar{\gamma}_{t}(X) d \alpha_{t}(X)=\iint_{\mathbb{R}^{3} \times \Omega} \xi(X) \cdot y d \gamma_{t}(X, y)
$$

for all continuous $\xi: \mathbb{R}^{3} \rightarrow \mathbb{R}^{3}$ of at most quadratic growth. Since $\chi$ is absolutely continuous with respect to $\mathcal{L}^{3}$, we deduce

$$
\int_{\mathbb{R}^{3}} \xi(X) \cdot \bar{\gamma}_{t}(X) d \alpha_{t}(X)=\int_{\Omega} \xi\left(\nabla P_{t}(x)\right) \cdot x d x .
$$

To justify generalizing (4) to (9), let us assume (1) has a smooth, stable solution $(P, u)$. Set $Y:=\nabla P$ and compute, for any $\zeta \in C_{c}^{\infty}\left(\mathbb{R}^{3}\right)$,

$$
\begin{aligned}
\frac{\mathrm{d}}{\mathrm{d} t} \int_{\mathbb{R}^{3}} \zeta(X) d \alpha_{t}(X) & =\int_{\Omega} \partial_{t} Y_{t}(x) \cdot \nabla \zeta\left(Y_{t}(x)\right) d x \\
& =-\int_{\Omega}\left[(u(t, x) \cdot \nabla) Y_{t}(x)\right] \cdot \nabla \zeta\left(Y_{t}(x)\right) d x+\int_{\Omega} J\left[Y_{t}(x)-x\right] \cdot \nabla \zeta\left(Y_{t}(x)\right) d x .
\end{aligned}
$$


Since $u$ is divergence-free and $\nabla Y_{t}=\nabla^{2} P_{t}$ is symmetric, we deduce that the first integral in the right hand side of the second equation of the above display is

$$
\begin{aligned}
\int_{\Omega}\left[\left(\nabla Y_{t}(x)\right)^{t} u(t, x)\right] \cdot \nabla \zeta\left(Y_{t}(x)\right) d x & =-\int_{\Omega}\left[\left(\nabla Y_{t}(x)\right)^{t} \nabla \zeta\left(Y_{t}(x)\right)\right] \cdot u(t, x) d x \\
& =-\int_{\Omega} \nabla\left[\zeta\left(Y_{t}(x)\right)\right] \cdot u(t, x) d x=0 .
\end{aligned}
$$

As for the second integral, we have, due to $J^{t}=-J, Y_{t \#} \chi=\alpha_{t}$ and (11),

$$
\begin{aligned}
\int_{\Omega} J\left[Y_{t}(x)-x\right] \cdot \nabla \zeta\left(Y_{t}(x)\right) d x & =-\int_{\Omega} Y_{t}(x) \cdot(J \nabla \zeta)\left(Y_{t}(x)\right) d x+\int_{\Omega} x \cdot(J \nabla \zeta)\left(Y_{t}(x)\right) d x \\
& =\int_{\mathbb{R}^{3}}(J X) \cdot \nabla \zeta(X) d \alpha_{t}(X)-\int_{\mathbb{R}^{3}}\left[J \bar{\gamma}_{t}(X)\right] \cdot \nabla \zeta(X) d \alpha_{t}(X) .
\end{aligned}
$$

Thus,

$$
\frac{\mathrm{d}}{\mathrm{d} t} \int_{\mathbb{R}^{3}} \zeta(X) d \alpha_{t}(X)=\int_{\mathbb{R}^{3}} J\left[X-\bar{\gamma}_{t}(X)\right] \cdot \nabla \zeta(X) d \alpha_{t}(X)
$$

which implies

$$
\partial_{t} \alpha(t, X)+\nabla_{X} \cdot(J[X-\bar{\gamma}(t, X)] \alpha(t, X))=0
$$

is satisfied in the sense of distributions.

Existence of a solution for the dual problem (2), (3), (9), (5) for any probability measure $\alpha_{0}$ with finite second moment was proved in [4].

In this paper we define weak Lagrangian solutions in physical space for any convex initial data $P_{0}$ (i.e. any initial probability measure $\alpha_{0}=\nabla P_{0 \#} \chi$ ), and prove existence of such solutions in the case of discrete measures. Moreover, we show conservation of geostrophic energy along the Lagrangian trajectories in the physical space.

One stand-out feature of Lagrangian solutions in physical space in the case of singular initial data is the absence of the Lebesgue measure preserving property for the flow map $F_{t}$, given in (ii) of Definition 1.1. Heuristically, since the solution of the dual problem (2), (3), (9), (5) does not provide enough information to separate the particle paths (in physical space) within the subgradient set $\partial P_{t}^{*}(X) \subset \Omega$ for $X \in \operatorname{supp} \alpha_{t}$, we define the flow map $F_{t}$ which collapses the set $\partial P_{t}^{*}(X)$ to one point, its " $\alpha_{t}$-barycenter" $\bar{\gamma}_{t}(X) \in \partial P_{t}^{*}(X)$, and thus the $\chi$-measure carried by the set $\partial P_{t}^{*}(X)$ should now be concentrated at the point $\bar{\gamma}_{t}(X)$. Then the measure preserving property $F_{t \#} \chi=\chi$ is replaced by

$$
F_{t \#} \chi=\mu_{t} \text { for } t>0, \text { where } \mu_{t}:=\left(\bar{\gamma}_{t} \circ \nabla P_{t}\right)_{\#} \chi \text {. }
$$

Note that in the case when $\alpha_{t}$ is absolutely continuous with respect to the Lebesgue measure, we have $\left(\bar{\gamma}_{t}(X) \circ \nabla P_{t}\right)(x)=\left(\nabla P_{t}^{*} \circ \nabla P_{t}\right)(x)=x$ for a.e. $x \in \Omega$, and thus we recover the Lebesgue measure preserving property of $F_{t}$ given in (ii) of Definition 1.1. We justify this definition of $F_{t}$ in the case of singular data, and its generalized measure-preserving property (14), by giving, in Section 4.1 below, an explicit example of a sequence of Lagrangian solutions $\left(P^{(i)}, F^{(i)}\right)$ in the sense of Definition 1.1 with absolutely continuous initial measures $\alpha_{0}^{i}:=\nabla P_{0 \#}^{(i)} \chi$ in the dual space, such that the sequence $\left(P^{(i)}, F^{(i)}\right)$ converges weakly to a Lagrangian solution $(P, F)$ in the sense of Definition 4.12 with a singular initial measure $\nabla P_{0 \#} \chi=\delta_{z_{0}}$. We show that this limiting solution $(P, F)$ satisfies the (generalized) measure-preserving property (14), yet does not satisfy (ii) of Definition 1.1. Thus, Definition 4.12 appears to be a natural extension of Definition 1.1 
to the case of singular initial data if we expect to have stability of weak Lagrangian solutions in physical space. We note that in the case of Lagrangian solutions with absolutely continuous initial measure $\alpha_{0}=\nabla P_{0 \#} \chi$, a stability property was proved in [14]. For solutions with singular initial data $\alpha_{0}$, stability is not proved at present, although the example described above suggests that some stability properties may be expected in this case too.

The rest of the paper is organized as following. In the next section we collect some results on the existence and properties of solutions in dual space. In Section 3 we prove that, under a mild time-regularity assumption, Eulerian solutions do not exist if the dual-space solutions have point masses at non-negligibly many times. This will advocate finding a suitable notion of weak Lagrangian solutions in physical case that makes sense in the general case of measurevalued solutions $\alpha$ in the dual space. This will be achieved in Section 4. There we will also prove that such solutions exist in the case where $\alpha_{t}$ are convex combinations of point masses, or equivalently, when $P_{0}$ is the maximum of finitely many affine functions. In Section 5 we will show that weak Lagrangian solutions in physical space can be translated into weak solutions of the problem in the dual space. This will lead to conservation of energy for weak Lagrangian solutions for the Semi-Geostrophic system.

\section{Solutions in dual space}

In this section we collect a number of results on the existence and some properties of solutions in dual space. Our main source is [4]. Before that, let us recall the definitions of some important objects. In the spirit of [5], one defines $A C^{p}\left(0, T ; \mathcal{P}_{2}\left(\mathbb{R}^{3}\right)\right)$ (for $\left.1 \leq p \leq \infty\right)$ as the set of all paths $\mu:[0, T] \ni t \rightarrow \mu_{t} \in \mathcal{P}_{2}\left(\mathbb{R}^{3}\right)$ for which there exists $\beta \in L^{p}(0, T)$ such that

$$
W_{2}\left(\mu_{s}, \mu_{t}\right) \leq \int_{s}^{t} \beta(\tau) d \tau \text { for all } 0 \leq s \leq t \leq T,
$$

where $W_{2}$ is the quadratic Wasserstein distance [20]. The smallest of the functions $\beta$ satisfying the inequality above is called the metric derivative of the curve $\mu$, it is denoted by $\left|\mu^{\prime}\right|$ and it satisfies [5] that

$$
\left|\mu^{\prime}\right|(t)=\lim _{s \rightarrow t} \frac{W_{2}\left(\mu_{s}, \mu_{t}\right)}{|s-t|} \text { for a.e. } t \in(0, T) .
$$

There exists a Borel velocity $\mathbf{v}:(0, T) \times \mathbb{R}^{3} \rightarrow \mathbb{R}^{3}$ transporting $\mu$ in the sense of distributions, i.e.

$$
\partial_{t} \mu+\nabla_{x} \cdot(\mathbf{v} \mu)=0 \text { in } \mathcal{D}^{\prime}\left((0, T) \times \mathbb{R}^{3}\right),
$$

such that $\mathbf{v}(t, \cdot) \in L^{2}\left(\mu_{t} ; \mathbb{R}^{3}\right)$ and $\left\|\mathbf{v}_{t}\right\|_{L^{2}\left(\mu_{t} ; \mathbb{R}^{3}\right)}=\left|\mu^{\prime}\right|(t)$ for a.e. $t \in(0, T)$. It turns out that this velocity (called "of minimal norm") minimizes $\left\|\mathbf{v}_{t}\right\|_{L^{2}\left(\mu_{t} ; \mathbb{R}^{3}\right)}$ among all possible Borel velocities (i.e. satisfying (15)), and it can be uniquely selected $\left(\mu_{t}\right.$-a.e.) for a.e. $t \in(0, T)$ by requiring that it belong to the closure of $\nabla C_{c}^{\infty}\left(\mathbb{R}^{3}\right)$ in $L^{2}\left(\mu_{t} ; \mathbb{R}^{3}\right)$, which is denoted [5] by $\mathcal{T}_{\mu_{t}} \mathcal{P}_{2}\left(\mathbb{R}^{3}\right)$ and called the tangent space to $\mathcal{P}_{2}\left(\mathbb{R}^{3}\right)$ at $\mu_{t}$. Finally, if $F: \mathcal{P}_{2}\left(\mathbb{R}^{3}\right) \rightarrow \mathbb{R}$ is lower semicontinuos with respect to the topology induced by the Wasserstein distance $W_{2}$, then we can define the subdifferential of $F$ at some $\mu \in \mathcal{P}_{2}\left(\mathbb{R}^{3}\right)$ as the set of all $\xi \in L^{2}\left(\mu ; \mathbb{R}^{3}\right)$ such that

$$
F(\nu) \geq F(\mu)+\iint_{\mathbb{R}^{3} \times \mathbb{R}^{3}} \xi(x) \cdot(y-x) \gamma(d x, d y)+o\left(W_{2}(\mu, \nu)\right)
$$

for all $\nu \in \mathcal{P}_{2}\left(\mathbb{R}^{3}\right)$ and all the optimal transport plans [20] $\gamma$ between $\mu$ and $\nu$. This set is denoted by $\partial F(\mu)$ and its element of minimal $L^{2}\left(\mu ; \mathbb{R}^{3}\right)$-norm is denoted by $\nabla_{w} F(\mu)$ and called [4] the Wasserstein gradient of $F$ at $\mu$. 
Adapting to our context (by, for example, replacing $J$ by $J^{t}=-J$ which still satisfies the required orthogonality property in [4]), a Hamiltonian ODE solution/trajectory for the lower semicontinuous "Hamiltonian" $H: \mathcal{P}_{2}\left(\mathbb{R}^{3}\right) \rightarrow \mathbb{R}$ is defined as follows.

Definition 2.1. A curve $\mu \in A C^{1}\left(0, T ; \mathcal{P}_{2}\left(\mathbb{R}^{3}\right)\right)$ with the property that there exists a vector field $\mathbf{v}:(0, T) \times \mathbb{R}^{3} \rightarrow \mathbb{R}^{3}$ such that

$$
(0, T) \ni t \rightarrow\left\|\mathbf{v}_{t}\right\|_{L^{2}\left(\mu_{t} ; \mathbb{R}^{3}\right)} \text { belongs to } L^{1}(0, T)
$$

and

$$
\begin{aligned}
& \partial_{t} \mu-\nabla \cdot(J \mathbf{v} \mu)=0, \mu_{0}=\tilde{\mu} \text { as distributions, } \\
& \mathbf{v}_{t} \in \mathcal{T}_{\mu_{t}} \mathcal{P}_{2}\left(\mathbb{R}^{3}\right) \cap \partial H\left(\mu_{t}\right) \text { for a.e. } t \in(0, T),
\end{aligned}
$$

is called a solution of the Hamiltonian ODE associated to the Hamiltonian $H$, starting at $\tilde{\mu}$.

Fix $\nu \in \mathcal{P}_{2}\left(\mathbb{R}^{3}\right)$ and define

$$
H_{\nu}: \mathcal{P}_{2}\left(\mathbb{R}^{3}\right) \rightarrow \mathbb{R}, \quad H_{\nu}(\mu)=-\frac{1}{2} W_{2}^{2}(\mu, \nu),
$$

which is, obviously, continuous. Then $\partial H_{\nu}(\mu)$ consists of all functions of the type $\bar{\gamma}-\mathrm{Id}$, where $\gamma$ is any optimal plan between $\mu$ and $\nu[20]$, and $\bar{\gamma}$ is its barycentric projection onto $\mu$, i.e. given by (see, e.g. [5])

$$
\iint_{\mathbb{R}^{3} \times \mathbb{R}^{3}} y \cdot \xi(x) \gamma(d x, d y)=\int_{\mathbb{R}^{3}} \bar{\gamma}(x) \cdot \xi(x) \mu(d x)
$$

for all $\xi \in C\left(\mathbb{R}^{3} ; \mathbb{R}^{3}\right)$ of at most quadratic growth. In light of the fact that the $y$-marginal of $\gamma$ is $\nu$, the above definition implies $\|\bar{\gamma}\|_{L^{2}\left(\mu ; \mathbb{R}^{3}\right)} \leq\|\operatorname{Id}\|_{L^{2}\left(\nu ; \mathbb{R}^{3}\right)}$. Thus,

$$
W_{2}^{2}(\mu, \nu)=\int_{\mathbb{R}^{3}}|x|^{2} \mu(d x)+\int_{\mathbb{R}^{3}}|y|^{2} \nu(d y)-2 \int_{\mathbb{R}^{3}} x \cdot \bar{\gamma}(x) \mu(d x) \geq\|\bar{\gamma}-\operatorname{Id}\|_{L^{2}\left(\mu ; \mathbb{R}^{3}\right)}^{2}
$$

for all such optimal plans, which implies

$$
\left\|\nabla_{w} H_{\nu}(\mu)\right\|_{L^{2}\left(\mu ; \mathbb{R}^{3}\right)} \leq 1+\sqrt{-2 H_{\nu}(\tilde{\mu})}=: a(\tilde{\mu}) \text { whenever } W_{2}(\mu, \tilde{\mu}) \leq 1 .
$$

Then one combines Lemma 7.6 [4] and Theorem 7.4 [4] to obtain on $[0,1 / a(\tilde{\mu})=: T]$ a solution $\mu \in A C^{\infty}\left(0, T ; \mathcal{P}_{2}\left(\mathbb{R}^{3}\right)\right)$ (Lipschitz curve) of the Hamiltonian ODE starting at $\tilde{\mu}$ and satisfying that it is $a(\tilde{\mu})$-Lipschitz and conservative, i.e. $[0, T] \ni t \rightarrow H_{\nu}\left(\mu_{t}\right)$ is constant. Thus, $a\left(\mu_{T}\right)=$ $a(\tilde{\mu})$, which means that $(20)$ is also satisfied with the same bounds if we replace $\tilde{\mu}$ by $\mu_{T}$. Thus, in light of the same results from [4], we infer that we can extend the solution to $[T, 2 T]$ (whereas it preserves the Lipschitz bound and conserves the Hamiltonian). By induction, we obtain:

Theorem 2.2. For any $\tilde{\mu} \in \mathcal{P}_{2}\left(\mathbb{R}^{3}\right)$ there exists a solution $\mu \in A C^{\infty}\left(0, \infty ; \mathcal{P}_{2}\left(\mathbb{R}^{3}\right)\right)$ of $(17)$ and (18), which starts at $\tilde{\mu}$, conserves $[0, \infty) \ni t \rightarrow H_{\nu}\left(\mu_{t}\right)$, and is globally a $(\tilde{\mu})-$ Lipschitz.

Let us now specialize to the case $\nu=\chi$ (defined in the introduction) and denote

$$
H: \mathcal{P}_{2}\left(\mathbb{R}^{3}\right) \rightarrow \mathbb{R}, \quad H(\mu)=-\frac{1}{2} W_{2}^{2}(\mu, \chi) .
$$

The absolute continuity of $\chi$ with respect to $\mathcal{L}^{3}$ adds the benefit of the fact that there is a unique optimal plan now between $\mu$ and $\chi$ for every $\mu \in \mathcal{P}_{2}\left(\mathbb{R}^{3}\right)$, namely $\gamma_{\mu}=(\nabla \Phi \times \mathrm{Id})_{\#} \chi$, where $\nabla \Phi$ is the optimal map pushing $\chi$ forward to $\mu$. Thus, we have

$$
\partial H(\mu) \cap \mathcal{T}_{\mu} \mathcal{P}_{2}\left(\mathbb{R}^{3}\right)=\left\{\bar{\gamma}_{\mu}-\mathrm{Id}\right\}=\left\{\nabla_{w} H(\mu)\right\} \text { for all } \mu \in \mathcal{P}_{2}\left(\mathbb{R}^{3}\right) .
$$

This means that Theorem 2.2 has the following: 
Corollary 2.3. Let $\alpha_{0} \in \mathcal{P}_{2}\left(\mathbb{R}^{3}\right)$ be given. Then there exists a distributional solution $\alpha \in$ $A C^{\infty}\left(0, \infty ; \mathcal{P}_{2}\left(\mathbb{R}^{3}\right)\right)$ for $(13)$ with $\alpha(0)=\alpha_{0}$, where $\bar{\gamma}_{t}$ is the barycentric projection of the optimal plan between $\alpha_{t}$ and $\chi$. In other words, the curve $\alpha$ is globally Lipschitz and satisfies the system (2), (3), (9), (5) in the sense of distributions in $(0, \infty) \times \mathbb{R}^{3}$. Also, the Hamiltonian energy $t \rightarrow H\left(\alpha_{t}\right)$ is conserved.

Remark 2.4. Since $[0, \infty) \ni t \rightarrow \alpha_{t} \in \mathcal{P}_{2}\left(\mathbb{R}^{3}\right)$ is continuous, we use Proposition 3.2 [19] to conclude that there is a family $P \in C\left([0, \infty) ; H^{1}(\Omega)\right)$ of convex functions $P(t, \cdot)$ such that $\nabla_{x} P(t, \cdot)=: \nabla P_{t}$ pushes $\chi$ forward to $\alpha_{t}$ optimally for all $t \geq 0$.

\section{Lagrangian vs Eulerian}

As of this date, the only weak Lagrangian solutions in the physical space have been shown to exist in the case $\nabla P_{0 \#} \chi=: \alpha_{0} \in L^{1}(\Omega)$. This is achieved in [14] by improving the $L^{q}(q>1)$ result in [9]. The solution $(P, F)$ constructed in these references satisfies $\nabla P_{t \#} \chi=: \alpha_{t} \in L^{q}(\Omega)$ for almost all $t \in(0, T)$. We work with solutions that may not satisfy any of these conditions, i.e. the measures $\alpha_{t}$ (for $t \in[0, T)$ ) may be singular. In particular, $\alpha_{t}$ can be Dirac measures $\delta_{z(t)}$ as in the example given in [14] (see also Section 4.1 below).

An interesting question is existence of Eulerian solutions in physical space. Recently, existence of such solutions for a class of initial data, where the conditions include the requirement that the support of the function $\alpha_{0}=\nabla P_{0 \#} \chi$ in the dual space be the whole space, was obtained by L. Ambrosio, M. Colombo, G. De Philippis, A. Figalli in two-dimensional periodic case [2] and in three-dimensional case with $\Omega=\mathbb{R}^{3}$ [3], based on the results of G. De Philippis and A. Figalli [13] on the regularity of solutions for the Monge-Ampere equation. Existence of Eulerian solutions in physical space for more general initial data $\alpha_{0} \in L^{q}(\Omega)$, when the support of $\alpha_{0}$ in the dual space may have a non-empty boundary, or if $\alpha_{0}$ is a singular measure, is presently not known.

In this section, we show that Eulerian solutions in the physical space exhibiting some mild regularity in time cannot give rise to "very irregular" solutions in dual space. Let us begin by recalling the definition of a weak solution of (1).

Definition 3.1. Let $u:[0, T) \times \Omega \rightarrow \mathbb{R}^{3}$ and $P:[0, T) \times \Omega \rightarrow \mathbb{R}$ satisfy $u \in L^{1}\left([0, T) \times \Omega ; \mathbb{R}^{3}\right)$, $\nabla P \in L^{\infty}([0, T) \times \Omega) \cap C\left([0, T) ; L^{1}(\Omega)\right)$, and $P(t, \cdot)$ is convex in $\Omega$ for every $t \in[0, \infty)$. The pair $(P, u)$ is a weak Eulerian solution of (1) if

$$
\begin{aligned}
& \int_{0}^{T} \int_{\Omega}\left\{\nabla P(t, x) \cdot\left[\partial_{t} \phi(t, x)+\nabla \phi(t, x) u(t, x)\right]\right. \\
& \quad+J[\nabla P(t, x)-x] \cdot \phi(t, x)\} d x d t+\int_{\Omega} \nabla P_{0}(x) \cdot \phi(0, x) d x=0
\end{aligned}
$$

for any $\phi \in C_{c}^{1}\left([0, T) \times \Omega ; \mathbb{R}^{3}\right)$, and

$$
\int_{0}^{T} \int_{\Omega} u(t, x) \cdot \nabla \psi(t, x) d x d t=0
$$

for any $\psi \in C_{c}^{1}([0, T) \times \bar{\Omega})$.

In what follows, we show that one cannot have weak Eulerian solutions exhibiting mild time regularity and spatial "flat parts" except, possibly, at negligibly many times. We begin with 
Proposition 3.3 below, whose proof is a relatively straight-forward adaptation of Corollary 2.3 and Proposition 2.4 in [19]. The only difference is that in the said reference the set $\mathcal{O}$ is a subset of $(0, T) \times \Omega$ of full measure.

Before stating the result, we need some preliminary observations. If $X \in H^{1}\left(0, T ; L^{2}\left(\Omega ; \mathbb{R}^{3}\right)\right)$, we denote by $\dot{X} \in L^{2}\left(0, T ; L^{2}\left(\Omega ; \mathbb{R}^{3}\right)\right)$ its functional derivative, defined by

$$
\lim _{h \rightarrow 0}\left\|\frac{X_{t+h}-X_{t}}{h}-\dot{X}_{t}\right\|_{L^{2}\left(\Omega ; R^{3}\right)}=0 \quad \text { for } \quad \mathcal{L}^{1}-\text { a.e. } \quad t \in(0, T) .
$$

In the next lemma, we extend $X$ to a map in $A C^{2}\left(\mathbb{R} ; L^{2}\left(\Omega ; \mathbb{R}^{3}\right)\right)$ by setting $X_{t}=X_{0+}$ for $t \leq 0$ and $X_{t}=X_{T-}$ for $t \geq T$.

Lemma 3.2. Let $X \in H^{1}\left(0, T ; L^{2}\left(\Omega ; \mathbb{R}^{3}\right)\right)$ and $\dot{X}$ be its functional derivative. Then

$$
\lim _{h \rightarrow 0} \int_{0}^{T} \int_{\Omega}\left|\frac{X_{t+h} x-X_{t} x}{h}-\dot{X}_{t} x\right|^{2} d x d t=0 .
$$

As a consequence, there exist sequences $h_{k}^{+} \rightarrow 0^{+}, h_{k}^{-} \rightarrow 0^{-}$and a measurable subset $\mathcal{A} \subset \mathbb{R} \times \Omega$ such that $\mathcal{L}^{4}((\mathbb{R} \times \Omega) \backslash \mathcal{A})=0$ and

$$
\lim _{k \rightarrow \infty} \frac{X_{t+h_{k}^{+}} x-X_{t} x}{h_{k}^{+}}=\lim _{k \rightarrow \infty} \frac{X_{t+h_{k}^{-}} x-X_{t} x}{h_{k}^{-}}=\dot{X}_{t} x
$$

for all $(t, x) \in \mathcal{A}$.

The proof in [15] needs no modification. The philosophy behind this result is that, in some specified sense, $\dot{X}$ can be viewed as almost a classical pointwise time-derivative of $X$. Also, since $X \in H^{1}\left(0, T ; L^{2}\left(\Omega ; \mathbb{R}^{3}\right)\right)$, we have that it admits a Borel representative. Equation (24) shows that $\dot{X}$ itself has that property. Throughout the paper we identify both $X$ and $\dot{X}$ with their Borel representatives.

Proposition 3.3. Let $X \in H^{1}\left(0, T ; L^{2}\left(\Omega ; \mathbb{R}^{3}\right)\right)$ be such that $X_{t}=\nabla P_{t}$, where $P_{t}$ is convex for all $t \in(0, T)$. Let $\mathcal{A} \subset(0, T) \times \Omega$ as in Lemma 3.2. Furthermore, let $\mathcal{O} \subset \mathcal{A}$ be a Borel set with $\mathcal{L}^{4}(\mathcal{O})>0$ and such that $\mathcal{L}^{3}\left(\left[X_{t} x\right]\right)>0$ for all $(t, x) \in \mathcal{O}$, where $\left[X_{t} x\right]:=\left\{y \in \Omega: X_{t} y=X_{t} x\right\}$. Then, there exists a Borel map $\mathbf{w}:(0, T) \times \mathbb{R}^{3} \rightarrow \mathbb{R}^{3}$ such that

$$
\dot{X}(t, x)=\mathbf{w}(t, X(t, x)) \text { for } \mathcal{L}^{4}-\text { a.e. }(t, x) \in \mathcal{O} .
$$

Proof: Let $\lambda$ denote the $\mathcal{L}^{4}$-measure restricted to $\mathcal{O}, \Psi: \mathcal{O} \rightarrow(0, T) \times \mathbb{R}^{3}$ given by $\Psi(t, x)=$ $(t, X(t, x))$, and set $\vartheta:=\Psi_{\#} \lambda$. Denote by $\eta$ the vector-measure whose density with respect to $\lambda$ is $\dot{X}$, then set $\sigma:=\Psi_{\#} \eta$. Clearly, $\sigma \ll \vartheta$, which means there exists a Borel vector field $\mathbf{w}:(0, T) \times \mathbb{R}^{3} \rightarrow \mathbb{R}^{3}$ such that $\mathrm{d} \sigma=\mathbf{w d} \vartheta$.

The disintegration theorem (see, for example, Theorem 5.3.1 [5]) applies to the Borel vector field $\Psi$ and the measure $\lambda$. Thus, for $\vartheta$-a.e. $(t, y) \in(0, T) \times \mathbb{R}^{3}$, there exists a unique Borel probability measure $\lambda_{t, y}$ on $\mathcal{O}$ such that the map $(t, y) \rightarrow \lambda_{t, y}(B)$ is Borel measurable for each Borel set $B \subset \mathcal{O}$. Furthermore, $\lambda_{t, y}\left(\Psi^{-1}(t, y)\right)=1$ for $\vartheta$-a.e. $(t, y) \in(0, T) \times \mathbb{R}^{3}$ and

$$
\iint_{\mathcal{O}} f(t, x) d \lambda(t, x)=\int_{0}^{T} \int_{\mathbb{R}^{3}}\left(\int_{\Psi^{-1}(t, y)} f(t, x) \lambda_{t, y}(d t, d x)\right) \vartheta(d t, d y)
$$


for every Borel measurable $f:(0, T) \times \Omega \rightarrow[0, \infty]$. We showed in [19], Theorem 2.2, that

$$
\mathbf{w}(t, X(t, x))=\int_{\Psi^{-1}(t, X(t, x))} \dot{X}(t, z) d \lambda_{t, X(t, x)}(t, z) \text { for } \lambda \text { - a.e. }(t, x) \in \mathcal{O} .
$$

Note that $(t, z) \in \Psi^{-1}(t, X(t, x))$ is equivalent to $(t, z) \in \mathcal{O}$ and $X(t, z)=X(t, x)$, so we have $\Psi^{-1}(t, X(t, x))=\mathcal{O} \cap\left[X_{t} x\right]$. Then we apply Proposition 2.4 in [19] to get that $\dot{X}(t, x)=\dot{X}(t, z)$ for all $(t, z) \in \mathcal{O} \cap\left[X_{t} x\right]$. According to (27), we get(26).

QED.

Before proving the main theorem of this section, we need a measurability lemma.

Lemma 3.4. Let $\alpha \in A C^{2}\left(0, T ; \mathcal{P}_{2}\left(\mathbb{R}^{3}\right)\right)$ for some $T>0$. Then the set

$$
\mathcal{D}(\alpha):=\left\{(t, X) \in(0, T) \times \mathbb{R}^{3}: X \text { is an atom of } \alpha_{t}\right\}
$$

is Borel.

Proof: Denote by $C_{c}^{+}$the nonnegative cone of $C_{c}\left(\mathbb{R}^{3}\right)$. Consider, for every positive integer $m$ and every $\xi \in C_{c}^{+}$, the set

$$
\mathcal{D}_{m}^{\xi}:=\left\{(t, X) \in(0, T) \times \mathbb{R}^{3}: \int_{\mathbb{R}^{3}} \xi(Y) d \alpha_{t}(Y) \geq \frac{1}{m} \xi(X)\right\} .
$$

Note that the absolute continuity in time of the left hand side of the above inequality [5] and the continuity in $X$ of the right hand side imply that the difference is a continuous function of $(t, X)$. Therefore, $\mathcal{D}_{m}^{\xi}$ is the nonnegative set of a continuous function, which makes it a closed subset of $(0, T) \times \mathbb{R}^{3}$. Thus,

$$
\mathcal{D}_{m}:=\left\{(t, X) \in(0, T) \times \mathbb{R}^{3}: \int_{\mathbb{R}^{3}} \xi(Y) d \alpha_{t}(Y) \geq \frac{1}{m} \xi(X) \text { for all } \xi \in C_{c}^{+}\right\}
$$

is closed as well, by being an arbitrary intersection of closed sets. Since

$$
\mathcal{D}(\alpha)=\cup_{m \geq 1} \mathcal{D}_{m},
$$

the proof is concluded.

QED.

Now we prove the main result of this section: that weak (Eulerian) solutions in the physical space exhibiting some mild regularity in time cannot give rise to "very irregular" solutions in dual space.

Theorem 3.5. Let $(P, u)$ be a weak solution for the Semi-Geostrophic system in the physical space such that $\nabla P \in H^{1}\left(0, T ; L^{2}\left(\Omega ; \mathbb{R}^{3}\right)\right)$. Then $\alpha_{t}:=\nabla P_{t \#} \chi$ is atom-free for $\mathcal{L}^{1}-a . e . t \in$ $(0, T)$.

Proof: Set $\Psi(t, x):=(t, X(t, x))$. Since $\Psi$ is a Borel map on $(0, T) \times \Omega$, due to Lemma 3.4 we infer that

$$
\mathcal{O}:=\Psi^{-1}(\mathcal{D}(\alpha)) \text { is a Borel subset of }(0, T) \times \Omega \text {. }
$$

One can see that

$$
\mathcal{O}=\left\{(t, x) \in(0, T) \times \Omega: \mathcal{L}^{3}\left(\left[X_{t} x\right]\right)>0\right\} .
$$


By Fubini's Theorem and by the convexity of the potentials whose gradients push $\chi$ forward to $\alpha_{t}$, we infer that there exists a Borel subset $\mathcal{T} \subset(0, T)$ such that

$$
\mathcal{O}=\cup_{t \in \mathcal{T}}\left(\{t\} \times \mathcal{O}_{t}\right),
$$

where $\mathcal{O}_{t}$ is the union of all (at most countably many) convex subsets of $\Omega$ of positive $\mathcal{L}^{3}$ measure on each of which $X_{t}$ is constant. Assume by contradiction that $\mathcal{L}^{1}(\mathcal{T})>0$. We further throw out of $\mathcal{T}$ the $\mathcal{L}^{1}$-negligible set of times at which

$$
\int_{\Omega} \nabla \zeta(x) u(t, x) d x \neq \mathbf{0} \in \mathbb{R}^{3}
$$

for all $\zeta \in C_{c}^{\infty}\left(\Omega ; \mathbb{R}^{3}\right)$ (via the separability of this space with respect to the sup norm), but we keep the notation $\mathcal{T}$ for the remaining subset. Consider $\mathcal{A}$ as the Borel subset of $(0, T) \times \Omega$ of full measure defined in Lemma 3.2, i.e. where the time pseudo-derivative of $X$ in the sense of (25) exists. The set $\mathcal{A} \cap \mathcal{O}$ is a Borel set (which we still denote by $\mathcal{O}$ ) with $\mathcal{L}^{4}(\mathcal{O})>0$. According to Proposition 3.3, we infer there exists a Borel map w : $(0, T) \times \mathbb{R}^{3} \rightarrow \mathbb{R}^{3}$ such that (26) is satisfied. By taking $\phi(t, x)=\xi(t) \zeta(x)$ with $\xi \in C_{c}^{\infty}(0, T)$ and $\zeta \in C_{c}^{\infty}\left(\Omega ; \mathbb{R}^{3}\right)$ in $(22)$ we discover that

$$
t \rightarrow \int_{\Omega} X(t, x) \cdot \zeta(x) d x \text { is absolutely continuous }
$$

and

$$
\frac{d}{d t} \int_{\Omega} X(t, x) \cdot \zeta(x) d x=\int_{\Omega}\{X(t, x) \cdot[\nabla \zeta(x) u(t, x)]+J[X(t, x)-x] \cdot \zeta(x)\} d x
$$

for a.e. $t \in(0, T)$ and every $\zeta \in C_{c}^{\infty}\left(\Omega ; \mathbb{R}^{3}\right)$ (via the usual argument involving the separability of this space endowed with the sup-norm). Throwing out, if necessary, a negligible set of times, we conclude

$$
\int_{\Omega} \dot{X}(t, x) \cdot \zeta(x) d x=\int_{\Omega}\{X(t, x) \cdot[\nabla \zeta(x) u(t, x)]+J[X(t, x)-x] \cdot \zeta(x)\} d x
$$

for a.e. $t \in(0, T)$ and every $\zeta \in C_{c}^{\infty}\left(\Omega ; \mathbb{R}^{3}\right)$. Choose such a $t_{0}$ that also lies in $\mathcal{T}$ and consider now only test functions $\zeta \in C_{c}^{\infty}\left(\omega_{0} ; \mathbb{R}^{3}\right)$, where $\omega_{0}$ is a connected component of $\mathcal{O}_{t_{0}}$ (which is a convex subset of $\Omega$ of positive $\mathcal{L}^{3}$-measure on which $X_{t_{0}}$ is constant). Since $X_{t_{0}} \equiv \mathbf{c} \in \mathbb{R}^{3}$ in $\omega_{0}$, we infer

$$
\left.\mathbf{w}\left(t_{0}, \mathbf{c}\right) \cdot \int_{\omega_{0}} \zeta(x) d x=\mathbf{c} \cdot \int_{\omega_{0}} \nabla \zeta(x) u(t, x) d x+\int_{\omega_{0}} J[\mathbf{c}-x] \cdot \zeta(x)\right\} d x
$$

for all $\zeta \in C_{c}^{\infty}\left(\omega_{0} ; \mathbb{R}^{3}\right)$. Due to (30) the first term in the right hand side vanishes and since the equality holds for all $\zeta \in C_{c}^{\infty}\left(\omega_{0} ; \mathbb{R}^{3}\right)$, we deduce

$$
\mathbf{w}\left(t_{0}, \mathbf{c}\right)=J[\mathbf{c}-x] \text { for a.e. } x \in \omega_{0},
$$

which contradicts the fact that $\omega_{0}$ has nonempty interior.

QED.

Remark 3.6. Thus, in order to accommodate singular solutions in dual space, we see the need for defining Lagrangian solutions instead of Eulerian ones in the physical space. Whereas solutions in the dual space may come in any form or shape (from pure Dirac deltas to functions), only the absolutely continuous ones with respect to the Lebesgue measures have been so far known to give rise to Lagrangian solutions in the physical space [9], [14]. In the next section we discuss an extension to this notion and prove some existence results. 


\section{Weak Lagrangian solutions in physical space for the case of singular initial data}

In [9], Lagrangian solutions in the physical space with initial data $\nabla P_{0 \#} \chi=\alpha_{0} \in L^{p}\left(\mathbb{R}^{3}\right)$ $(p>1)$, were constructed by the following procedure. First, time-stepping approximation from [10] combined with results of [1] yield existence of a solution $(\alpha, P)$ of the dual space system (2)-(4) with initial data (5), and a locally bounded map $\Phi:(0, T) \times \mathbb{R}^{3} \rightarrow \mathbb{R}^{3}$ satisfying

$$
\dot{\Phi}=U(\cdot, \Phi), \mathcal{L}^{4}-\text { a.e. in }(0, T) \times \nabla P_{0}(\Omega), \quad \Phi(0, X)=X \text { for } \alpha_{0}-\text { a.e. } X \in \mathbb{R}^{3},
$$

such that

$$
\alpha_{t}=\Phi_{t \#} \alpha_{0} \quad \text { for all } t>0 .
$$

There also exists a Borel map $\Phi^{*}$ such that $\Phi_{t}^{*}$ preserve $\mathcal{L}^{3}$ and $\Phi_{t} \circ \Phi_{t}^{*}=\operatorname{Id}=\Phi_{t}^{*} \circ \Phi_{t}$ a.e. in $\mathbb{R}^{3}$. The physical space flow is defined as

$$
F_{t}:=\nabla P_{t}^{*} \circ \Phi_{t} \circ \nabla P_{0} .
$$

Then it is shown that $(P, F)$ satisfies all the requirements of Definition 1.1.

In order to see what can be expected in the case of general initial data, we consider an example in which $\alpha_{0}$ is a point mass.

\subsection{An example: a limit of Lagrangian solutions, with initial measures weakly converging to a point mass}

We discuss the case $\alpha_{0}=\frac{4}{3} \pi \delta_{z_{0}}$ for some $z_{0} \in \mathbb{R}^{3}$ by considering a sequence of approximations

$$
\alpha_{0}^{(\varepsilon)}=\frac{1}{\varepsilon^{3}} \chi_{B_{z_{0}}(\varepsilon)}, \quad \text { where } \varepsilon>0
$$

where $\chi_{A}$ denotes the indicator function of the set $A$. This is a version of the counterexample in [14]. For notational simplicity, we drop the requirements that $\alpha_{t}$ be probability measures and that $\mathcal{L}^{3}(\Omega)=1$; thus, $\alpha_{t}$ are measures of total mass equal to $\mathcal{L}^{3}(\Omega)$. We fix the domain in physical space to be the ball $\Omega=B_{1}(0) \subset \mathbb{R}^{3}$, which means $\chi=\left.\mathcal{L}^{3}\right|_{B_{1}(0)}$. It is easy to see that $\alpha_{0}=\nabla P_{0 \#} \chi$ and $\alpha_{0}^{(\varepsilon)}=\nabla P_{0 \#}^{(\varepsilon)} \chi$, where

$$
\begin{aligned}
& P_{0}(x)=z_{0} \cdot x, \\
& P_{0}^{(\varepsilon)}(x)=z_{0} \cdot x+\varepsilon \frac{|x|^{2}}{2} .
\end{aligned}
$$

Following the calculations in [14], we find that for each $\varepsilon>0$, one Lagrangian solution $\left(P^{(\varepsilon)}, F^{(\varepsilon)}\right)$ in the sense of Definition 1.1 with initial data $P_{0}^{(\varepsilon)}$, can be obtained as follows. Let $z(t)$ be determined by solving $\dot{z}(t)=J z(t), z(0)=z_{0}$; thus, $z(t)=L_{1}(t) z_{0}$, where

$$
L_{c}(t)=\left(\begin{array}{rrr}
\cos c t & -\sin c t & 0 \\
\sin c t & \cos c t & 0 \\
0 & 0 & 1
\end{array}\right) \quad \text { for } c \in \mathbb{R} .
$$

Then

$$
\begin{aligned}
& P_{t}^{(\varepsilon)}(x)=z(t) \cdot x+\varepsilon \frac{|x|^{2}}{2}, \\
& F_{t}^{(\varepsilon)}(x)=L_{c}(t) x, \text { with } c=1-\frac{1}{\varepsilon} .
\end{aligned}
$$


We also note that the Legendre transform (over $\Omega$ ) of $P_{t}^{(\varepsilon)}$ is

$$
\left(P_{t}^{(\varepsilon)}\right)^{*}(X)=\left\{\begin{array}{cl}
\frac{|X-z(t)|^{2}}{2 \varepsilon}, & \text { if } X \in B_{z(t)}(\varepsilon) ; \\
|X-z(t)|-\frac{\varepsilon}{2}, & \text { if } X \notin B_{z(t)}(\varepsilon),
\end{array}\right.
$$

and the flow map in the dual variables is

$$
\Phi_{t}^{(\varepsilon)}(X)=z(t)+M_{c}(t)\left(X-z_{0}\right), \text { with } c=1-\frac{1}{\varepsilon},
$$

where

$$
M_{c}=\left(\begin{array}{rrr}
\cos c t & -\sin c t & 0 \\
\sin c t & \cos c t & 0 \\
0 & 0 & 0
\end{array}\right)
$$

Expression (35) was obtained by (32) using $\left(P_{t}^{(\varepsilon)}\right)^{*}, \Phi_{t}^{(\varepsilon)}$ given above.

Now we take limits as $\varepsilon \rightarrow 0+$. Clearly,

$$
\nabla P_{t}^{(\varepsilon)} \rightarrow \nabla P_{t} \quad \text { uniformly in } \Omega \quad \text { for all } t \geq 0,
$$

where

$$
P_{t}(x)=z(t) \cdot x \quad \text { for } \quad x \in \Omega, t \geq 0 .
$$

Note that by taking $t=0$ in (38) we obtain the function $P_{0}$ in (33). On the other hand, as far as $F_{t}^{(\varepsilon)}$ is concerned, we see that

$$
F_{0}^{(\varepsilon)}(x)=x \text { for all } x \in \Omega, \varepsilon>0,
$$

yet, for any $t>0$ the sequence $F_{t}^{(\varepsilon)}(\cdot)$ does not converge even in a weak sense. Then we consider a limit of $F^{(\varepsilon)}$ as functions of $(t, x)$, and we note that, as $\varepsilon \rightarrow 0+$,

$$
F^{(\varepsilon)} \rightarrow 0 \quad \text { weakly in } L^{2}\left([0, T] \times \Omega ; \mathbb{R}^{3}\right) \text { for any } T>0 .
$$

(39) determines the limit $F^{(\varepsilon)}$ only up to the set of points $(t, x)$ of $\mathcal{L}^{4}$-measure zero. However, since the initial measure $\alpha_{0}$ for the limiting problem is a point mass, it is natural to expect that the flow map for such problem is sensitive to changes on sets of $\mathcal{L}^{4}$-measure zero. In particular, we cannot take the flow map of the form $F \equiv 0$ for the limiting problem, since we require $F(0, \cdot) \equiv$ Id. Thus, in the following lemma we note some properties which are satisfied by any map $\hat{F}:[0, T) \times \Omega \rightarrow \Omega$ which is a weak limit of $F^{(\varepsilon)}$. We first note the following: for $P_{t}$ in (38) we have

$$
\alpha_{t}:=\nabla P_{t \#} \chi=\frac{4}{3} \pi \delta_{z(t)},
$$

and the Kantorovich optimal plan $\gamma_{t}:=\left(\nabla P_{t} \times \mathrm{Id}\right)_{\#} \chi$ is defined by $\gamma_{t}(A \times B)=\delta_{z(t)}(A) \chi(B)$ for all Borel $A \subset \mathbb{R}^{3}, B \subset \Omega$. Then (10) implies that the barycentric projection of $\gamma_{t}$ onto $\alpha_{t}$ is

$$
\bar{\gamma}_{t}(z(t))=0,
$$

which defines $\bar{\gamma}_{t}(X)$ for $\alpha_{t}$-a.e. $X \in \mathbb{R}^{3}$. 
Lemma 4.1. Let $T>0$ and $\hat{F}:[0, T) \times \Omega \rightarrow \Omega$ be a Borel map such that

$$
F^{(\varepsilon)} \rightarrow \hat{F} \quad \text { weakly in } L^{2}\left([0, T] \times \Omega ; \mathbb{R}^{3}\right) \text { for any } T>0
$$

as $\epsilon \rightarrow 0^{+}$, where $F^{(\varepsilon)}$ is defined by (35). Then

i. $\hat{F}_{t \#} \chi=\frac{4}{3} \pi \delta_{0} \quad$ for a.e. $t>0$. In particular, the map $\hat{F}_{t}$ does not preserve $\left.\mathcal{L}^{3}\right|_{\Omega}$ for a.e. $t$.

ii. $(P, \hat{F})$ satisfies property (14) for a.e. $t>0$. Here $P$ is defined by (38).

Proof: Assertion (i) follows from (39) and Fubini Theorem.

To show (ii), we fix any $t>0$ and note that (38), (40) imply $\bar{\gamma}_{t}\left(\nabla P_{t}(x)\right)=\bar{\gamma}_{t}(z(t))=0$ for every $x \in \Omega$, which implies $\left(\bar{\gamma}_{t} \circ \nabla P_{t}\right)_{\#} \chi=\frac{4}{3} \pi \delta_{0}$. Now (ii) follows from (i).

QED.

Remark 4.2. Lemma 4.1 suggests that for the case of singular initial measure $\alpha_{0}$, the $\mathcal{L}^{3}$ measure preserving property of $F_{t \#} \chi=\chi$ of $F_{t}$ should be replaced by (14) if we expect to have stability of weak Lagrangian solutions in physical space.

Now we discuss a possible construction of a Lagrangian solution in physical space for the limiting initial data $P_{0}(x)=z_{0} \cdot x$, i.e. with $\alpha_{0}=\frac{4}{3} \pi \delta_{z_{0}}$, and compare it with the limit of $\left(P^{(\varepsilon)}, F^{(\varepsilon)}\right)$ obtained above. We use $P_{t}$ in (38) to see that $\alpha_{t}:=\nabla P_{t \#} \chi=\frac{4}{3} \pi \delta_{z(t)}$. One can readily check that the property $\dot{z}(t)=J z(t), z(0)=z_{0}$ implies that $\alpha$ is a weak solution of the $\mathrm{SG}$ in dual space, i.e. of the problem (2), (3), (9), (5).

In order to construct a flow map $F_{t}$ in in physical space, let us go back to the definition of $F_{t}=\nabla P_{t}^{*} \circ \Phi_{t} \circ \nabla P_{0}$ from [9]. Since in the general case (when $\alpha_{t}$ is a measure) the place of $\nabla P_{t}^{*}$ is taken by the barycentric projection $\bar{\gamma}_{t}$ (see (13)), it is natural to ask if one can obtain a solution by putting

$$
\begin{aligned}
& F_{0} \equiv \mathrm{Id}, \\
& F_{t}:=\bar{\gamma}_{t} \circ \Phi_{t} \circ \nabla P_{0} \quad \text { for } t>0 .
\end{aligned}
$$

Of course, for this function to be well-defined, one needs the Lagrangian flow in dual space $\Phi_{t}$ to be defined at $z_{0}$ for all $t \in[0, T]$. Thus, we solve

$$
\dot{\Phi}(t, X)=J\left[\Phi(t, X)-\bar{\gamma}_{t}(\Phi(t, X))\right] \text { with } \Phi(0, X)=X
$$

for $X=z_{0}$. Using (40) we rewrite this as

$$
\dot{\Phi}\left(t, z_{0}\right)=J \Phi\left(t, z_{0}\right) \text { with } \Phi\left(0, z_{0}\right)=z_{0} .
$$

Since $\dot{z}(t)=J z(t), z(0)=z_{0}$, then uniqueness for the Cauchy problem associated to this ODE system implies

$$
\Phi\left(t, z_{0}\right)=z(t) .
$$

Now, $F_{t}$ is well-defined by (41), and explicitly is

$$
F(t, x)=F_{t}(x)= \begin{cases}x, & \text { if } t=0, x \in \Omega \\ 0, & \text { if } t>0, x \in \Omega\end{cases}
$$


From (39), (43) we conclude that, as $\varepsilon \rightarrow 0+$,

$$
\begin{aligned}
& F^{(\varepsilon)} \rightarrow F \quad \text { weakly in } L^{2}\left([0, T] \times \Omega ; \mathbb{R}^{3}\right), \text { for any } T>0, \\
& F_{0}^{(\varepsilon)} \equiv F_{0} \equiv \operatorname{Id} \quad \text { in } \Omega, \quad \text { for any } \varepsilon>0 .
\end{aligned}
$$

We also notice that

$$
F_{t \#} \chi=\frac{4}{3} \pi \delta_{0} \text { for all } t>0 .
$$

Moreover, we showed in the proof of Lemma 4.1 that

$$
\left(\gamma_{t} \circ \nabla P_{t}\right)_{\#} \chi=\frac{4}{3} \pi \delta_{0} \quad \text { for } t>0 .
$$

Then (45) implies that $F_{t}$ satisfies the generalized measure-preserving property (14) for all $t>0$. Furthermore, since $\dot{z}(t)=J z(t), z(0)=z_{0}$, and $Z(t, x):=\left(\nabla P_{t}\right)(F(t, x))=z(t)$, it follows that $(P, F)$ satisfies equation $(7)$ in the sense $(8)$. Consequently, we have arrived to:

Lemma 4.3. Let $P$ be defined by (38). Then $F$ defined by (41) has the explicit form (43), and $(P, F)$ satisfies the following:

(a) $(P, F)$ is a weak limit (in the sense of (37), (44)) as $\varepsilon \rightarrow 0^{+}$, of a family of Lagrangian solutions $\left(P^{(\varepsilon)}, F^{(\varepsilon)}\right)$ in the sense of Definition 1.1;

(b) $(P, F)$ satisfies equation (7) in the sense (8);

(c) For all $t>0,(P, F)$ satisfies property (14), which takes the form (45).

In other words, the limit $(P, F)$ satisfies the properties of Lagrangian solution (as in Definition 1.1) except for the $\mathcal{L}^{3}$-measure preserving property of $F_{t}$, which is replaced by (14). An important remaining issue, the continuity of $t \rightarrow F_{t}(\cdot)$, will be clarified in the next section.

Remark 4.4. The above computations of the Lagrangian flow in dual space highlight the difficulty in applying the theory of regular Lagrangian flows [1] to the case of singular initial measures $\alpha_{0}$. One can extend $\bar{\gamma}_{t}$, defined by (40), to all $\mathbb{R}^{3}$ by using that $P_{t}^{*}(X)=|X-z(t)|$ is differentiable for $X \neq z(t)$, defining

$$
\bar{\gamma}_{t}(X):=\nabla P_{t}^{*}(X)=\frac{X-z(t)}{|X-z(t)|} \text { if } X \neq z(t) .
$$

Then one can easily check that $U(t, X):=J\left[X-\bar{\gamma}_{t}(X)\right]$ satisfies the conditions in [1], therefore a unique solution to the $O D E$ (42) exists and is unique for Lebesgue-a.e. $X \in \mathbb{R}^{3}$ such that $\Phi_{t}$ preserves $\mathcal{L}^{3}$. However, when $\alpha_{0}$ is singular, the set $\left\{X \in \mathbb{R}^{3}: \Phi(t, X)\right.$ exists and is unique $\}$ may not contain all (if any) of the support of $\alpha_{0}$. In the case $\alpha_{0}=\delta_{z_{0}}$, the solution $\Phi\left(\cdot, z_{0}\right)$ can be found explicitly by solving the $O D E$ system, and this defines $\Phi(\cdot, X)$ for $\alpha_{0}$-a.e. $X \in \mathbb{R}$. In general, for singular measures $\alpha_{0}$, it is not clear how to find a suitable Lagrangian flow $\Phi(t, X)$ in the dual space that is defined for $\alpha_{0}$-a.e $X$.

\subsection{Definition of Lagrangian solutions with singular initial data, and exis- tence results}

In order to see the motivation for Definition 4.12 of Lagrangian solutions for the case of singular initial data given below, we first study properties of flows in physical space given by (41) in the 
case when $\alpha_{0}=\nabla P_{0 \#} \chi$ is possibly a singular measure, under the following assumptions:

(i) $P_{0} \in H^{1}(\Omega)$ is convex, $\alpha_{0}=\nabla P_{0 \#} \chi \in \mathcal{P}_{2}\left(\mathbb{R}^{3}\right)$;

(ii) There exists a Borel map $\Phi:[0, T] \times \mathbb{R}^{3} \rightarrow \mathbb{R}^{3}$ such that (42) holds in the integral sense for $\alpha_{0}$-a.e. $X \in \mathbb{R}^{3}$;

(iii) The family of measures $\alpha_{t}:=\Phi_{t \#} \alpha_{0}$ for $t \in[0, T)$ is a solution in $\mathcal{D}^{\prime}\left((0, T) \times \mathbb{R}^{3}\right)$ for the equation (2) with $U$ defined by (9), (10), where $\gamma_{t}$ is the unique optimal transport plan between $\alpha_{t}$ and $\chi$. This also defines a convex $P_{t}$ in $\Omega$ (for each $t$ ) such that $\gamma_{t}:=\left(\nabla P_{t} \times \mathrm{Id}\right)_{\#} \chi$;

(iv) $\alpha \in A C^{\infty}\left(0, \infty ; \mathcal{P}_{2}\left(\mathbb{R}^{3}\right)\right)$.

Note that assumptions (46) say that there exists a Lagrangian solution of the dual problem with initial data $P_{0}$. In the case when $P_{0} \in W^{1, \infty}(\Omega)$ and $\alpha_{0}=\nabla P_{0 \#} \chi \in L^{p}\left(\mathbb{R}^{3}\right)$ for $p \in(1, \infty]$ existence of $\Phi_{t}$ such that (46) is satisfied is shown in [9].

Below it is convenient to work with a Borel representative of the barycentric projection $\bar{\gamma}$. That is why we prove the following lemma:

Lemma 4.5. There exists a Borel measurable function defined on $(0, T) \times \mathbb{R}^{3}$ which for $\mathcal{L}^{1}$-a.e. $t \in(0, T)$ coincides with $\bar{\gamma}(t, X)$ for $\alpha(t, \cdot)$-a.e. $X \in \mathbb{R}^{3}$.

Proof: Let $\vartheta \in \mathcal{P}\left((0, T) \times \mathbb{R}^{3} \times \Omega\right)$ be the Borel probability given by

$$
\int_{0}^{T} \int_{\mathbb{R}^{3}} \int_{\Omega} \varphi(t, X, y) \vartheta(d t, d X, d y)=\frac{1}{T} \int_{0}^{T} \int_{\mathbb{R}^{3}} \int_{\Omega} \varphi(t, X, y) \gamma(t, d X, d y) d t
$$

for any continuous and bounded $\varphi$. Since $\alpha(t, \cdot)$ is the $X$-marginal of $\gamma(t, \cdot, \cdot)$, the $(t, X)$ marginal of $\vartheta$ is $\tilde{\vartheta} \in \mathcal{P}\left((0, T) \times \mathbb{R}^{3}\right)$ given by

$$
\int_{0}^{T} \int_{\mathbb{R}^{3}} \zeta(t, X) \tilde{\vartheta}(d t, d X)=\frac{1}{T} \int_{0}^{T} \int_{\mathbb{R}^{3}} \zeta(t, X) \alpha(t, d X) d t
$$

Thus, by disintegrating $\vartheta$ we get

$$
\int_{0}^{T} \int_{\mathbb{R}^{3}} \int_{\Omega} \varphi(t, X, y) \vartheta(d t, d X, d y)=\frac{1}{T} \int_{0}^{T} \int_{\mathbb{R}^{3}}\left(\int_{\Omega} \varphi(t, X, y) \vartheta(t, X ; d y)\right) \alpha(t, d X) d t,
$$

where $\vartheta(t, X ; \cdot)$ are Borel probabilities on $\Omega$ such that the map $(t, X) \rightarrow \vartheta(t, X ; B)$ is Borel for any Borel set $B \subset \Omega$. In particular, the maps

$$
(t, X) \rightarrow \int_{\Omega} \mathbf{f}(y) \vartheta(t, X ; d y)
$$

are Borel for all $\mathbf{f} \in C_{b}\left(\Omega ; \mathbb{R}^{3}\right)$. By taking $\varphi(t, X, y)=u(t) \xi(X) \cdot y$ and using (10), we conclude that

$$
\bar{\gamma}(t, X)=\int_{\Omega} y \vartheta(t, X ; d y) \text { for } \mathcal{L}^{1}-\text { a.e. } t \in(0, T) \text { and } \alpha(t, \cdot)-\text { a.e. } X \in \mathbb{R}^{3} .
$$

This finishes the proof.

QED. 
Remark 4.6. In light of Lemma 4.5 and equation (9), we see immediately that there exists a Borel measurable function defined on $(0, T) \times \mathbb{R}^{3}$ which for $\mathcal{L}^{1}$-a.e. $t \in(0, T)$ coincides with $U(t, X)$ for $\alpha(t, \cdot)$-a.e. $X \in \mathbb{R}^{3}$.

Remark 4.7. In view of the above lemma, from now on we shall use the notation $\bar{\gamma}$ to denote the Borel representative of the barycentric projection defined by (10). Likewise, by following the same proof, it is easy to see that the barycentric projection of $\left(\operatorname{Id} \times \nabla P_{t}\right)_{\#} \chi$ onto its first marginal (namely, $\chi$ ) can also be extended to a Borel map from $(0, T) \times \Omega$ into $\mathbb{R}^{3}$. Since this coincides with $\nabla P_{t}(x)$ for $\mathcal{L}^{4}$-a.e. $(t, x) \in(0, T) \times \Omega$, we shall assume in the remainder of the paper that $\nabla P$ is Borel measurable in both variables.

We also note the following:

Lemma 4.8. For $(t, X) \in[0, T) \times \mathbb{R}^{3}$ denote

$$
\Omega_{t, X}:=\left\{x \in \Omega: \nabla P_{t}(x) \text { exists and } \nabla P_{t}(x)=X\right\} .
$$

Then

$$
\bar{\gamma}_{t}(X) \in \Omega_{t, X} \text { for } \alpha(t, \cdot) \text {-a.e. } X \in \mathbb{R}^{3} \text {. }
$$

In particular,

$$
\left(\nabla P_{t} \circ \bar{\gamma}_{t}\right)(X)=X \text { for every } t \in[0, T) \text { and } \alpha(t, \cdot) \text {-a.e. } X \in \mathbb{R}^{3} .
$$

Proof: Fix $t \in[0, T)$. Since $\gamma_{t}=\left(\nabla P_{t} \times \mathrm{Id}\right)_{\#} \chi$ and $\pi_{1 \#} \gamma_{t}=\alpha_{t}$, by disintegrating $\gamma_{t}$ as in Theorem 5.3.1 [5], we get

$$
\gamma_{t}=\int_{\mathbb{R}^{3}} \gamma_{t, X} d \alpha_{t}(X)
$$

where $\gamma_{t, X}$ is a family of probability measures on $\Omega$ such that the map $X \rightarrow \gamma_{t, X}(B)$ is Borel for any Borel set $B \subset \Omega$, and

$$
\gamma_{t, X}\left(\mathbb{R}^{3} \backslash \Omega_{t, X}\right)=0 \text { for } \alpha(t, \cdot) \text {-a.e. } X \in \mathbb{R}^{3} .
$$

From (10) and the disintegration, we get

$$
\bar{\gamma}_{t}(X)=\int_{\Omega} y d \gamma_{t, X}(y)=\int_{\Omega_{t, X}} y d \gamma_{t, X}(y) \text { for } \alpha(t, \cdot) \text {-a.e. } X \in \mathbb{R}^{3} .
$$

The convexity of $P_{t}(\cdot)$ implies that $\Omega_{t, X}$ is a convex set (of dimension $k(t, X) \in\{0,1,2,3\}$ ) for every $X$ such that $\Omega_{t, X} \neq \emptyset$. Thus,

$$
\bar{\gamma}_{t}(X) \in \Omega_{t, X} \text { for } \alpha(t, \cdot) \text {-a.e. } X \in \mathbb{R}^{3},
$$

and for such $X$ we get $\left(\nabla P_{t} \circ \bar{\gamma}_{t}\right)(X)=X$.

QED.

The continuity of $F$ in time was proved in [9], [14] in the case of absolutely continuous $\alpha_{t}$. In general we cannot expect that, which is clear from the structure (41) of $F_{t}$, especially looking at the example when $P_{0}$ is linear, i.e. $\alpha_{0}=\delta_{z_{0}}$ discussed above: streamlines in the physical space are concentrated on the barycenters $\bar{\gamma}_{t}(X)$ of the sets $\partial P_{t}^{*}(X)$ where $X \in \operatorname{supp}\left(\alpha_{t}\right)$. Thus it is natural to expect continuity of $F_{t}$ "relative to $\nabla P_{t}$ ". Indeed, we have the following: 
Proposition 4.9. Assume that (46) hold. Then the map F defined in (41) satisfies

$$
\lim _{t \rightarrow t_{0}} \int_{\Omega} \xi\left(\nabla P_{t} \circ F_{t}(x)\right) \cdot F_{t}(x) d x=\int_{\Omega} \xi\left(\nabla P_{t_{0}} \circ F_{t_{0}}(x)\right) \cdot F_{t_{0}}(x) d x
$$

for all $t_{0} \in[0, \infty)$ (which we define as meaning $t \rightarrow 0^{+}$if $t_{0}=0$ ) and all $\xi \in C_{c}\left(\mathbb{R}^{3} ; \mathbb{R}^{3}\right)$.

Furthermore,

$$
\lim _{t \rightarrow t_{0}} \int_{\Omega} \xi\left(\nabla P_{t_{0}} \circ F_{t_{0}}(x)\right) \cdot F_{t}(x) d x=\int_{\Omega} \xi\left(\nabla P_{t_{0}} \circ F_{t_{0}}(x)\right) \cdot F_{t_{0}}(x) d x,
$$

and

$$
\lim _{t \rightarrow 0^{+}} \int_{\Omega} \xi\left(\nabla P_{0}(x)\right) \cdot F_{t}(x) d x=\int_{\Omega} \xi\left(\nabla P_{0}(x)\right) \cdot x d x
$$

for all $t_{0} \in[0, \infty)$ and all $\xi \in C_{c}\left(\mathbb{R}^{3} ; \mathbb{R}^{3}\right)$.

Proof: The (unique) optimal transport plan between its $X$-marginal $\alpha_{t}$ and it $y$-marginal $\chi$ is $\gamma_{t}=\left(\nabla P_{t} \times \mathrm{Id}\right)_{\#} \chi$. Let $t_{0} \in[0, \infty)$. Since $W_{2}\left(\alpha_{t}, \alpha_{t_{0}}\right) \rightarrow 0$ as $t \rightarrow t_{0}$ (which we define as meaning $t \rightarrow 0^{+}$if $t_{0}=0$ ), by stability of optimal plans [5], we infer that $\gamma_{t}$ converges to $\gamma_{t_{0}}$ in $\mathcal{P}_{2}\left(\mathbb{R}^{3} \times \Omega\right)$. We use the definition (41) of $F_{t}$, the fact that $\nabla P_{0 \#} \chi=\alpha_{0}$ and $\Phi_{t \#} \alpha_{0}=\alpha_{t}$, and (10) to get for $t>0$

$$
\int_{\Omega} \xi\left(\Phi_{t} \circ \nabla P_{0}(x)\right) \cdot F_{t}(x) d x=\iint_{\mathbb{R}^{3} \times \mathbb{R}^{3}} \xi(X) \cdot y \gamma_{t}(d X, d y) .
$$

For $t=0$, we use $F_{0}=\mathrm{Id}, \Phi_{0}=\mathrm{Id}, \nabla P_{0 \#} \chi=\alpha_{0}$ and (10) to get the equality displayed above. Now, by the continuity of $t \rightarrow \gamma_{t}$ proved above, we deduce for $t_{0} \in[0, \infty)$

$$
\lim _{t \rightarrow t_{0}} \int_{\Omega} \xi\left(\Phi_{t} \circ \nabla P_{0}(x)\right) \cdot F_{t}(x) d x=\int_{\mathbb{R}^{3}} \int_{\Omega} \xi(X) \cdot y \gamma_{t_{0}}(d X, d y)=\int_{\Omega} \xi\left(\Phi_{t_{0}} \circ \nabla P_{0}(x)\right) \cdot F_{t_{0}}(x) d x .
$$

Next we note that $\left(\Phi_{t} \circ \nabla P_{0}\right)_{\#} \chi=\alpha_{t}$. Combining this with (48), we obtain for every $t \in(0, T)$ :

$$
\nabla P_{t} \circ \bar{\gamma}_{t} \circ \Phi_{t} \circ \nabla P_{0}(x)=\Phi_{t} \circ \nabla P_{0}(x) \quad \text { for a.e. } x \in \Omega \text {. }
$$

Then using (41) we obtain for every $t \in(0, T)$

$$
\nabla P_{t} \circ F_{t}(x)=\Phi_{t} \circ \nabla P_{0}(x) \text { for a.e. } x \in \Omega \text {. }
$$

For $t=0$, we deduce (53) from $F_{0}=\mathrm{Id}, \Phi_{0}=\mathrm{Id}$. Now (52) implies (49).

Furthermore, (31) implies

$$
\Phi_{t}(X)=\Phi_{t_{0}}(X)+\int_{t_{0}}^{t} U\left(s, \Phi_{s}(X)\right) d s \text { for } \alpha_{0}-\text { a.e. } X \in \mathbb{R}^{3} \text { and all } 0 \leq t_{0} \leq t .
$$

This yields

$$
\begin{aligned}
\left|\xi\left(\Phi_{t}(X)\right)-\xi\left(\Phi_{t_{0}}(X)\right)\right|^{2} & \leq\|\nabla \xi\|_{\infty}^{2}\left(\int_{t_{0}}^{t}\left|U\left(s, \Phi_{s}(X)\right)\right| d s\right)^{2} \\
& \leq\left(t-t_{0}\right)\|\nabla \xi\|_{\infty}^{2} \int_{t_{0}}^{t}\left|U\left(s, \Phi_{s}(X)\right)\right|^{2} d s
\end{aligned}
$$


for $\alpha_{0}$-a.e. $X \in \mathbb{R}^{3}$. Note that $(0, T) \times \mathbb{R}^{3} \ni(t, X) \rightarrow U\left(t, \Phi_{t}(X)\right)$ is Borel (as composition of Borel maps). Next we use $\nabla P_{0 \#} \chi=\alpha_{0}$ and $\Phi_{s \#} \alpha_{0}=\alpha_{s}$ to get

$$
\left\|\xi \circ \Phi_{t} \circ \nabla P_{0}-\xi \circ \nabla \Phi_{t_{0}} \circ \nabla P_{0}\right\|_{L^{2}\left(\Omega ; R^{3}\right)} \leq \sqrt{t-t_{0}}\|\nabla \xi\|_{\infty}\left(\int_{t_{0}}^{t} \int_{\mathbb{R}^{3}}|U(s, Y)|^{2} d \alpha_{s}(Y) d s\right)^{1 / 2} .
$$

Then, using (53) we obtain

$$
\left\|\xi \circ \nabla P_{t} \circ F_{t}-\xi \circ \nabla P_{t_{0}} \circ F_{t_{0}}\right\|_{L^{2}\left(\Omega ; \mathbb{R}^{3}\right)} \leq \sqrt{t-t_{0}}\|\nabla \xi\|_{\infty}\left(\int_{t_{0}}^{t} \int_{\mathbb{R}^{3}}|U(s, Y)|^{2} d \alpha_{s}(Y) d s\right)^{1 / 2} .
$$

Since $\left\|U_{t}\right\|_{L^{2}\left(\alpha_{t} ; R^{3}\right)} \in L^{\infty}(0, \infty)$ and $F$ is bounded uniformly in time-space, we use (49) and the inequality displayed above to deduce (50). For $t_{0}=0$ we use the fact that $F_{0}(x)=x$ for a.e. $x$ to get (51) from (50).

QED.

Remark 4.10. In the case $\alpha_{t} \ll \mathcal{L}^{3}$ and $1<p<\infty$, note that the strong $L^{p}$-continuity in time $\left\|F_{t}-F_{t_{0}}\right\|_{L^{p}(\Omega)} \rightarrow 0$ as $t \rightarrow t_{0}$ is equivalent to weak $L^{p}$-continuity, since all the maps $F_{t}$

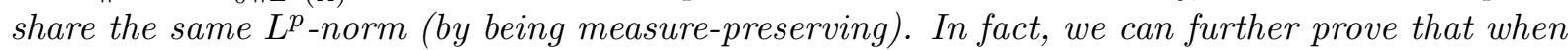
$\alpha_{t} \ll \mathcal{L}^{3}$ for all $t \geq 0$, (50) is equivalent to this weak $L^{p}$-continuity. Thus, our result above simply generalizes the $L^{p}$-continuity obtained in [9].

Proof: Let $1<q<\infty$ be such that $1 / p+1 / q=1$. First, one can easily check that

$$
\zeta \in L^{q}\left(\alpha_{t_{0}} ; \mathbb{R}^{3}\right) \Leftrightarrow \zeta \circ \Phi_{t_{0}} \circ \nabla P_{0} \in L^{q}\left(\Omega ; \mathbb{R}^{3}\right), \xi \in L^{q}\left(\Omega ; \mathbb{R}^{3}\right) \Leftrightarrow \xi \circ \nabla P_{0}^{*} \circ \Phi_{t_{0}}^{*} \in L^{q}\left(\alpha_{t_{0}} ; \mathbb{R}^{3}\right) .
$$

Thus, pick $\xi \in L^{q}\left(\Omega ; \mathbb{R}^{3}\right)$ and a sequence $\left\{\xi_{n}\right\}_{n} \subset C_{c}^{1}\left(\mathbb{R}^{3} ; \mathbb{R}^{3}\right)$ which converges to $\xi \circ \nabla P_{0}^{*} \circ \Phi_{t_{0}}^{*}$ strongly in $L^{q}\left(\alpha_{t_{0}} ; \mathbb{R}^{3}\right)$, i.e. $\xi_{n} \circ \Phi_{t_{0}} \circ \nabla P_{0}$ converges strongly to $\xi$ in $L^{q}\left(\Omega ; \mathbb{R}^{3}\right)$. We have

$\left|\int_{\Omega} \xi \cdot\left(F_{t}-F_{t_{0}}\right) d x\right| \leq\left|\int_{\Omega} \xi_{n} \circ \Phi_{t_{0}} \circ \nabla P_{0} \cdot\left(F_{t}-F_{t_{0}}\right) d x\right|+C(p, \Omega)\left\|\xi_{n} \circ \Phi_{t_{0}} \circ \nabla P_{0}-\xi\right\|_{L^{q}\left(\Omega ; R^{3}\right)}$.

We choose $n_{0}$ sufficiently large such that the last term in the right hand side is sufficiently small. Then use (50) for $\xi=\xi_{n_{0}}$ to conclude that (50) implies the weak $L^{p}$-continuity for $t \rightarrow F_{t}$ at $t_{0}$. A similar argument can be used to prove the converse.

QED.

Proposition 4.9 motivates the following

Definition 4.11. Let $P:[0, \infty) \times \Omega \rightarrow \mathbb{R}$ be such that $P \in C\left([0, \infty) ; H^{1}(\Omega)\right)$ and $P(t, \cdot)$ is convex in $\Omega$ for each $t \in[0, \infty)$. A map $F:[0, \infty) \times \Omega \rightarrow \Omega$ is called weakly $P$-continuous if (50), (51) hold for all $t_{0} \in(0, \infty)$ and all $\xi \in C_{c}\left(\mathbb{R}^{3} ; \mathbb{R}^{3}\right)$.

Furthermore, we note that considering the example of initial data in the dual space being a linear combination of Dirac masses (see also Proposition 4.14 below), the solution $\alpha_{t}$ at each time $t$ is also a linear combination of Dirac masses concentrated in the time-dependent location, it is not clear whether distinct initial location of Dirac masses should imply that their locations are distinct at all times. Thus we cannot expect existence of the map $F^{*}$ as in Definition 1.1(iii).

In light of the above, we are ready to generalize Definition 1.1 as follows: 
Definition 4.12. Let $P:[0, \infty) \times \Omega \rightarrow \mathbb{R}$ be such that $P \in C\left([0, \infty) ; H^{1}(\Omega)\right)$ and $P(t, \cdot)$ is convex in $\Omega$ for each $t \in[0, \infty)$. Let $F:[0, \infty) \times \Omega \rightarrow \Omega$ be a weakly $P$-continuous Borel map. Denote by $\bar{\gamma}_{t}$ the barycentric projection of the measure $\gamma_{t}:=\left(\nabla P_{t} \times \mathrm{Id}\right)_{\#} \chi$, defined in (10), and set

$$
\alpha_{t}:=\nabla P_{t \#} \chi, \quad \mu_{t}:=\bar{\gamma}_{t \#} \alpha_{t} .
$$

Then the pair $(P, F)$ is called a weak Lagrangian solution of $(1)$ in $[0, T) \times \Omega$ if

i. $F(0, x)=x$ and $P(0, x)=P_{0}(x)$ for all $x \in \Omega$;

ii. for any $t>0$ the mapping $F_{t}=F(t, \cdot): \Omega \rightarrow \Omega$ satisfies $F_{t \#} \chi=\mu_{t}$ and $F_{t \#} \mu_{0}=\mu_{t}$;

iii. The function $Z:(0, T) \times \Omega \rightarrow \mathbb{R}^{3}$ defined by

$$
Z_{t}=\nabla P_{t} \circ F_{t}
$$

lies, along with $F$, in $L^{\infty}\left(0, T ; L^{2}\left(\Omega ; \mathbb{R}^{3}\right)\right)$ and is a distributional solution of (7) in the sense of (8).

The following proposition gives sufficient conditions for the existence of weak Lagrangian solutions in physical space. Note that these conditions are exactly what was proved in [9] in the case $\alpha_{t} \in L^{q}\left(\mathbb{R}^{3}\right)$.

Proposition 4.13. Assume that (46) hold. Define $F$ by (41). Then the pair $(P, F)$ is a weak Lagrangian solution in physical space in the sense of Definition 4.12.

Furthermore, if there exists a Borel map $\Phi^{*}:[0, T] \times \mathbb{R}^{3} \rightarrow \mathbb{R}^{3}$ such that $\Phi_{\#}^{*} \alpha_{t}=\alpha_{0}$ for all $t \in[0, T)$, then

$$
\begin{aligned}
& \text { there exists a Borel mapping } F^{*}:[0, T] \times \Omega \rightarrow \Omega \text { satisfying } \\
& F_{t \#}^{*} \mu_{t}=\mu_{0} \text { and } F_{t}^{*} \circ F_{t}=\operatorname{Id} \mu_{0} \text {-a.e., and } F_{t} \circ F_{t}^{*}=\operatorname{Id} \mu_{t}-a . e .
\end{aligned}
$$

Proof: Proposition 4.9 implies that $F$ is weakly $P$-continuous.

From (48) and since $\nabla P_{t \#} \chi=\alpha_{t}$, we have:

$$
\nabla P_{t} \circ \bar{\gamma}_{t} \circ \nabla P_{t}(x)=\nabla P_{t}(x) \text { for every } t \in(0, T) \text { and } \chi-\text { a.e. } x \in \Omega \text {. }
$$

Now, in order to prove (i) of Definition 4.12 for $F$, we find that

$$
\bar{\gamma}_{0} \circ\left(\Phi_{0} \circ \nabla P_{0} \circ \bar{\gamma}_{0}\right) \circ \nabla P_{0}(x)=\bar{\gamma}_{0} \circ \nabla P_{0}(x) \text { for } \chi \text {-a.e. } x \in \Omega,
$$

due to (58) for $t=0$ and the hypothesis on $\Phi_{0}$. Since $\mu_{0}=\bar{\gamma}_{0} \circ \nabla P_{0 \#} \chi$, then (i) follows.

To prove (ii) we check that for $t>0$

$$
F_{t \#} \chi=\left(\bar{\gamma}_{t} \circ \Phi_{t} \circ \nabla P_{0}\right)_{\#} \chi=\left(\bar{\gamma}_{t} \circ \Phi_{t}\right)_{\#} \alpha_{0}=\bar{\gamma}_{t \#} \alpha_{t}=\mu_{t},
$$

where we used the definitions (55), and that $\Phi_{t \#} \alpha_{0}=\alpha_{t}$. Similarly, for $t>0$

$$
\begin{aligned}
F_{t \#} \mu_{0} & =\left(\bar{\gamma}_{t} \circ \Phi_{t} \circ \nabla P_{0}\right)_{\#} \mu_{0} \\
& =\left(\bar{\gamma}_{t} \circ \Phi_{t} \circ \nabla P_{0} \circ \bar{\gamma}_{0}\right)_{\#} \alpha_{0} \\
& =\left(\bar{\gamma}_{t} \circ \Phi_{t}\right)_{\#} \alpha_{0} \\
& =\bar{\gamma}_{t \#} \alpha_{t}=\mu_{t},
\end{aligned}
$$


where we used in the second line that $\left(\nabla P_{0} \circ \bar{\gamma}_{0}\right)_{\#} \alpha_{0}=\alpha_{0}$ by (48) for $t=0$.

Next we prove (iii) of Definition 4.12. We first note that $Z$ and $F$ are Borel (therefore, Lebesgue) measurable as compositions of Borel maps (see Lemma 4.5). By the definition of $\bar{\gamma}$, we have

$$
\begin{aligned}
\left|\int_{\mathbb{R}^{3}} \xi(X) \cdot \bar{\gamma}_{t}(X) d \alpha_{t}(X)\right| & \leq\left(\int_{\mathbb{R}^{3}}|\xi(X)|^{2} d \gamma_{t}(X, y)\right)^{1 / 2}\left(\int_{\Omega}|y|^{2} d \gamma_{t}(X, y)\right)^{1 / 2} \\
& =\|\xi\|_{L^{2}\left(\alpha_{t} ; \mathbb{R}^{3}\right)}\left(\int_{\Omega}|y|^{2} d y\right)^{1 / 2} .
\end{aligned}
$$

It follows that for all $t \in(0, T)$ we have

$$
\bar{\gamma}_{t} \in L^{2}\left(\alpha_{t} ; \mathbb{R}^{3}\right) \text { with }\left\|\bar{\gamma}_{t}\right\|_{L^{2}\left(\alpha_{t} ; \mathbb{R}^{3}\right)} \leq R_{0}\left(\mathcal{L}^{3}(\Omega)\right)^{1 / 2},
$$

where $0<R_{0}<\infty$ is large enough such that $\Omega \subset B\left(0, R_{0}\right)$. Thus, there exists $C \in \mathbb{R}$ independent of $t$ such that

$$
\int_{\Omega}\left|F_{t}(x)\right|^{2} d x=\int_{\mathbb{R}^{3}}\left|\bar{\gamma}_{t} \circ \Phi_{t}\right|^{2} d \alpha_{0}=\int_{\mathbb{R}^{3}}\left|\bar{\gamma}_{t}\right|^{2} d \alpha_{t}=\left\|\bar{\gamma}_{t}\right\|_{L^{2}\left(\alpha_{t} ; \mathbb{R}^{3}\right)}^{2} \leq C .
$$

Also, using that $F_{t \#} \chi=\mu_{t}$ as proved above, and also using (48), we have for $Z_{t}=\nabla P_{t} \circ F_{t}$ :

$$
\begin{gathered}
\int_{\Omega}\left|Z_{t}(x)\right|^{2} d x=\int_{\Omega}\left|\nabla P_{t}(y)\right|^{2} d \mu_{t}(y)=\int_{\Omega}\left|\nabla P_{t}\left(\bar{\gamma}_{t}(X)\right)\right|^{2} d \alpha_{t}(X) \\
=\int_{\mathbb{R}^{3}}|X|^{2} d \alpha_{t}(X)=\int_{\Omega}\left|\nabla P_{t}(x)\right|^{2} d x \leq \tilde{C}
\end{gathered}
$$

where $\tilde{C}<\infty$ is a constant coming from the fact that $P \in C\left([0, T] ; H^{1}(\Omega)\right)$. Thus, both $Z$ and $F$ belong to $L^{\infty}\left(0, T ; L^{2}\left(\Omega ; \mathbb{R}^{3}\right)\right)$. Next we note that assumption (ii) of (46) implies that for for $\chi$-a.e. $x \in \Omega$, the function $\dot{\Phi}\left(\cdot, \nabla P_{0}(x)\right)$ is a weak solution of the problem

$$
\dot{\Phi}\left(t, \nabla P_{0}(x)\right)=J\left[\Phi\left(t, \nabla P_{0}(x)\right)-\bar{\gamma}_{t}\left(\Phi\left(t, \nabla P_{0}(x)\right)\right)\right] \quad \text { with } \quad \Phi\left(0, \nabla P_{0}(x)\right)=\nabla P_{0}(x) .
$$

From (53), for each $t \in[0, T)$ the equality $Z_{t}(x)=\Phi_{t} \circ \nabla P_{0}$ holds for a.e. $x \in \Omega$. Thus using the integrability of $Z$ and $F$ proved above, using a function $\varphi \in C_{0}^{1}\left([0, T) \times \mathbb{R}^{3}\right)$ in the weak form of (60) and integrating with respect to $x$, we get (8).

Finally, in order to prove (57) under the additional assumption of the existence of $\Phi^{*}$, we set

$$
F_{0}^{*}(x)=x, \quad F_{t}^{*}(x):=\bar{\gamma}_{0} \circ \Phi_{t}^{*} \circ \nabla P_{t} \text { for } t>0 .
$$

Then property (57) is obvious for $t=0$. Thus we fix $t>0$ and and compute

$$
F_{t \#}^{*} \mu_{t}=\left(\bar{\gamma}_{0} \circ \Phi_{t}^{*} \circ \nabla P_{t} \circ \bar{\gamma}_{t}\right)_{\#} \alpha_{t}=\left(\bar{\gamma}_{0} \circ \Phi_{t}^{*}\right)_{\#} \alpha_{t}=\bar{\gamma}_{0 \#} \alpha_{0}=\mu_{0} .
$$

To finish proving (57), note that $F_{t}^{*} \circ F_{t}(y)=y$ for $\mu_{0}$-a.e. $y \in \Omega$ amounts to

$$
F_{t}^{*} \circ F_{t} \circ \bar{\gamma}_{0} \circ \nabla P_{0}(x)=\bar{\gamma}_{0} \circ \nabla P_{0}(x)
$$

for $\chi$-a.e. $x \in \Omega$. Since

$$
\left(F_{t}^{*} \circ F_{t} \circ \bar{\gamma}_{0}\right)\left(\nabla P_{0}(x)\right)=\left(\bar{\gamma}_{0} \circ \Phi_{t}^{*} \circ \nabla P_{t} \circ \bar{\gamma}_{t} \circ \Phi_{t} \circ \nabla P_{0} \circ \bar{\gamma}_{0}\right)\left(\nabla P_{0}(x)\right)
$$


and $\nabla P_{t} \circ \bar{\gamma}_{t}, \nabla P_{0} \circ \bar{\gamma}_{0}, \Phi_{t}^{*} \circ \Phi_{t}$ are all equal to the identity on the corresponding domains, we deduce (62).

QED.

In the case of discrete measures we can prove that the construction works. Even though not explicitly present in [4], a simple argument inserted in the proof of Theorem 7.4 from said reference yields that the solutions to the Hamiltonian ODE constructed there are convex combinations of point masses provided that the initial measures are of the same form (coefficients of the convex combinations are time-invariant). Our Hamiltonian in (21) satisfies all the requirements for Theorem 7.4 [4] to apply (see Lemma $7.6[4]$ ).

Proposition 4.14. Let $\mathbf{x}_{\mathbf{0}}=\left(x_{0}^{1}, \ldots, x_{0}^{n}\right) \in \mathbb{R}^{3 n}$ be arbitrary for some integer $n \geq 1$, and let

$$
\bar{\mu}=\sum_{i=1}^{n} c_{i} \delta_{x_{0}^{i}}
$$

be a convex combination of the Diracs at these points, i.e. nonnegative constants $c_{i}$ satisfy $\sum_{i=1}^{n} c_{i}=1$. Then the solution constructed in Theorem 7.4 [4] for the initial-value problem associated to the Hamiltonian ODE as in Definition 2.1 for the Hamiltonian in (21) is of the form

$$
\alpha_{t}=\sum_{i=1}^{n} c_{i} \delta_{x^{i}(t)}
$$

where $[0, T] \ni t \rightarrow \mathbf{x}(t)$ is in $W^{1, \infty}\left(0, T ; \mathbb{R}^{3 n}\right)$ and $\mathbf{x}(0)=\mathbf{x}_{0}$.

Proof: Let $m$ be a positive integer and set $h=T / m$. Then take $w_{0}^{m}:=-J \nabla H(\bar{\alpha})$, where $\nabla H(\alpha)$ denotes the element of $\partial H(\alpha)$ with least $L^{2}\left(\alpha ; \mathbb{R}^{3}\right)$-norm, and set, for all $t \in[0, h]$,

$$
\alpha_{t}^{m}=\left(\operatorname{Id}+t w_{0}^{m}\right)_{\#} \bar{\alpha}, \nu_{t}^{m}=\left(\operatorname{Id}+t w_{0}^{m}\right)_{\#}\left(w_{0}^{m} \bar{\alpha}\right) \text { and } w_{t}^{m}:=\frac{\mathrm{d} \nu_{t}^{m}}{\mathrm{~d} \alpha_{t}^{m}},
$$

where we used the fact (see Lemma $7.1[4]$ ) that $\nu_{t}^{m} \ll \alpha_{t}^{m}$ to get the Radon-Nykodim derivative $w_{t}^{m}$. On the next time subinterval $[h, 2 h]$ one defines $\alpha_{t}^{m}$ and $w_{t}^{m}$ similarly by using $\alpha_{h}^{m}$ and $w_{h}^{m}$ instead of $\bar{\alpha}$ and $w_{0}^{m}$, and $t-h$ instead of $t$. In general, the construction can be extended to $[k h,(k+1) h]$ for $k=0, \ldots, m-1$ by using $\alpha_{k h}^{m}$ and $w_{k h}^{m}, t-k h$ instead of $t$, and repeating the steps above. It is proved in [4] that the paths of measures $t \rightarrow \alpha_{t}^{m}$ are uniformly bounded in $\mathcal{P}_{2}\left(\mathbb{R}^{3}\right)$ and uniformly Lipschitz continuous. For a subsequence $m_{j} \rightarrow \infty$ we have a limiting $t \rightarrow \alpha_{t}$, which is shown to satisfy the Hamiltonian ODE with $\alpha_{0}=\bar{\alpha}$. Since

$$
\alpha_{t}^{m_{j}}=\left[\mathrm{Id}+(t-k h) w_{k h}^{m_{j}}\right]_{\#} \alpha_{k h}^{m_{j}}
$$

for $t \in[k h,(k+1) h]$, we deduce that all probabilities $\alpha_{t}^{m_{j}}$ are convex combinations of $n$ Dirac masses if $\alpha_{k h}^{m_{j}}$ is (with same coefficients). This is true for all $k=0, \ldots, m_{j}-1$, so we deduce that it holds for $\alpha_{t}^{m_{j}}$ all $t \in[0, T]$. The uniform bounds on $\alpha_{t}^{m}$ mentioned above translate into the uniform $L^{\infty}\left(0, T ; \mathbb{R}^{3 n}\right)$ bounds on $t \rightarrow \mathbf{x}^{m_{j}}(t)=\left(x^{m_{j}, 1}(t), \ldots, x^{m_{j}, n}(t)\right)$ (where $x^{m_{j}, i}(t)$ are the points in the support of $\left.\alpha_{t}^{m_{j}}\right)$. Furthermore, the uniform Lipschitz continuity of the paths $t \rightarrow \alpha_{t}^{m}$ in the Wasserstein space $\mathcal{P}_{2}\left(\mathbb{R}^{3}\right)$ gives a finite constant $C>0$ for which $W_{2}\left(\alpha_{t}^{m_{j}}, \alpha_{s}^{m_{j}}\right) \leq C|t-s|$ for all $t, s \in[0, T]$. Now fix $t_{0} \in(0, T)$. Note that $\mathbf{x}^{m}$ are piecewise linear and continuous in time, thus it is clear that

$$
C^{2}\left|t-t_{0}\right|^{2} \geq W_{2}^{2}\left(\alpha_{t}^{m_{j}}, \alpha_{t_{0}}^{m_{j}}\right)=\sum_{i=1}^{n} c_{i}\left|x^{m_{j}, i}(t)-x^{m_{j}, i}\left(t_{0}\right)\right|^{2} \text { for all } t \text { close enough to } t_{0} .
$$


Thus, the vector functions $t \rightarrow \mathbf{x}^{m_{j}}(t)$ are uniformly Lipschitz. By Ascoli-Arzela's theorem, a subsequence converges in the sup norm to a function $\mathbf{x} \in W^{1, \infty}\left(0, T ; \mathbb{R}^{3 n}\right)$, which implies the limiting measures $\alpha_{t}$ found above must have the form (64) for all $t \in[0, T]$. This follows from

$$
W_{2}^{2}\left(\alpha_{t}^{m_{j}}, \beta_{t}\right) \leq \sum_{i=1}^{n} c_{i}\left|x^{m_{j}, i}(t)-x^{i}(t)\right|^{2} \rightarrow 0 \text { as } j \rightarrow \infty,
$$

where $\beta_{t}$ is the convex combination of the Dirac masses at $x_{i}(t)$ with coefficients $c_{1}, \ldots, c_{n}$. QED.

So far we are not aware of any reason why distinct initial $x_{0}^{k}$ should give rise to distinct $x^{k}(t)$ at all later times. As a consequence, existence of the map $\Phi^{*}$ is uncertain (since transport maps from an average of $n$ points masses to one of $m$ point masses exist if and only if $n \geq m$ ).

Proposition 4.15. Let $n$ be a positive integer and

$$
\alpha_{t}:=\sum_{i=1}^{n} c_{i} \delta_{x^{i}(t)}, \text { for } t \in[0, T)
$$

be the solution of $S G$ in dual space constructed in Proposition 4.14 with initial data

$$
\alpha_{0}:=\sum_{i=1}^{n} c_{i} \delta_{x_{0}^{i}}
$$

where $x_{0}^{i}$ are arbitrary in $\mathbb{R}^{3}, i=1, \ldots, n$, and $c_{i}$ are nonnegative and $\sum_{i=1}^{n} c_{i}=1$. Then the map $\Phi^{n}:[0, T] \times \mathbb{R}^{3} \rightarrow \mathbb{R}^{3}$ given by $\Phi^{n}(t, X)=X$ if $X \neq x_{0}^{i}$, and $\Phi^{n}\left(t, x_{0}^{i}\right)=x^{i}(t)$ for $t \in[0, T]$, $i=1, \ldots, n$ satisfies $(46),($ ii $)-($ iii $)$.

Proof: It is obvious that $\Phi^{n}$ is Borel and $\Phi^{n}$ satisfies $\Phi_{t \#}^{n} \alpha_{0}=\alpha_{t}$. To show that $\Phi^{n}$ solves (42) we start from the fact that $\alpha_{t}$ solves the system (17), (18). In fact, the Hamiltonian (21) has the property that $\mathcal{T}_{\alpha} \mathcal{P}_{2}\left(\mathbb{R}^{3}\right) \cap \partial H(\alpha)=\{\bar{\gamma}-\mathrm{Id}\}$ (see, e.g. [4]), so $\alpha_{t}$ solves (13) in the sense of distributions. This is equivalent to

$$
t \rightarrow \int_{\mathbb{R}^{3}} \xi(X) d \alpha_{t}(X) \text { is absolutely continuous }
$$

and for a.e. $t \in(0, T)$ we have

$$
\frac{d}{d t} \int_{\mathbb{R}^{3}} \xi(X) d \alpha_{t}(X)=\int_{\mathbb{R}^{3}} \nabla \xi(X) \cdot U(t, X) d \alpha_{t}(X) \text { for all } \xi \in C_{c}^{1}\left(\mathbb{R}^{3}\right),
$$

for $U$ given in (9). According to Proposition 4.14, we have that $t \rightarrow x^{i}(t)$ is in $W^{1, \infty}\left(0, T ; \mathbb{R}^{3}\right)$ for all $i=1, \ldots, n$, which implies

$$
\sum_{i=1}^{n} c_{i} \nabla \xi\left(x^{i}(t)\right) \cdot \dot{x}^{i}(t)=\sum_{i=1}^{n} c_{i} \nabla \xi\left(x^{i}(t)\right) \cdot U\left(t, x^{i}(t)\right) \text { for all } \xi \in C_{c}^{1}\left(\mathbb{R}^{3}\right) .
$$

This leads to the desired conclusion.

QED.

We are now in a position to formulate: 
Corollary 4.16. Let $P_{0}$ be the maximum of finitely many affine functions: for some integer $n \geq 1$ and $a_{i} \in \mathbb{R}^{3}, b_{i} \in \mathbb{R}^{1}$ for $i=1, \ldots, n$

$$
P_{0}(x)=\max _{i=1, \ldots, n}\left(a_{i} \cdot x+b_{i}\right), \quad \text { for } x \in \Omega .
$$

Then there exists a weak Lagrangian solution for (1) in the sense of Definition 4.12 with $P(0, \cdot)=$ $P_{0}$ a.e. in $\Omega$.

\section{$5 \quad$ Return to dual space and conservation of energy}

We show that weak Lagrangian solutions give rise to solutions in dual space.

Theorem 5.1. Let $(P, F)$ be a weak Lagrangian solution of (1) in the sense of Definition 4.12 and set $\alpha_{t}:=\nabla P_{t \#} \chi$. Then $\alpha$ is a distributional solution of (13).

Proof: We need to show that for every $\varphi \in C_{0}^{\infty}\left((0, T) \times \mathbb{R}^{3}\right)$

$$
\int_{0}^{T} \int_{\mathbb{R}^{3}}\left(\partial_{t} \varphi+J\left[X-\bar{\gamma}_{t}(X)\right] \cdot \nabla \varphi\right) d \alpha_{t}(X) d t=0 .
$$

By the density argument, it is sufficient to show that for

$$
\varphi(t, X)=\zeta(t) \xi(X), \quad \text { for all } \xi \in C_{0}^{\infty}\left(\mathbb{R}^{3}\right), \zeta \in C_{0}^{\infty}(0, T) .
$$

Fix such $\xi, \zeta$.

From (55), $\left(\nabla P_{t} \circ \bar{\gamma}_{t}\right)_{\#} \alpha_{t}=\nabla P_{t \#} \mu_{t}$. Then (48) yields

$$
\nabla P_{t \#} \mu_{t}=\alpha_{t}
$$

Then $F_{t \#} \chi=\mu_{t}$ implies $Z_{t \#} \chi=\left(\nabla P_{t} \circ F_{t}\right)_{\#} \chi=\nabla P_{t \#} \mu_{t}=\alpha_{t}$, i.e.

$$
Z_{t \#} \chi=\alpha_{t} .
$$

Now we calculate using (66):

$$
\int_{0}^{T} \int_{\Omega} \zeta^{\prime}(t) \xi\left(Z_{t}(x)\right) d x d t=\int_{0}^{T} \int_{\mathbb{R}^{3}} \zeta^{\prime}(t) \xi(X) d \alpha_{t} d t .
$$

On the other hand, we can show that integrating by parts in $t$ and using the regularity of $Z, F$ and equation (8), we get

$$
\int_{0}^{T} \int_{\Omega} \zeta^{\prime}(t) \xi\left(Z_{t}(x)\right) d x d t=-\int_{0}^{T} \int_{\Omega} \zeta(t) \nabla \xi\left(Z_{t}(x)\right) \cdot J\left[Z_{t}(x)-F_{t}(x)\right] d x d t .
$$

Indeed, let $\eta^{\varepsilon}(t, x)$ be the family of standard mollifiers in time-space. We extend $Z(t, x)$ to $\mathbb{R}^{1} \times \mathbb{R}^{3}$ by defining it to be zero outside of $(0, T) \times \Omega$, and define $Z^{\varepsilon}=\eta^{\varepsilon} * Z(t, x)$ on $(0, T) \times \mathbb{R}^{3}$, where the convolution is with respect to $(t, x)$. Then $Z^{\varepsilon} \in C^{\infty}\left((0, T) \times \mathbb{R}^{3}\right)$. Also, (8) implies that the distributional derivative $\partial_{t} Z(t, x)$ in $(0, T) \times \mathbb{R}^{3}$ is $J(Z-F)$ (extended by zero outside of $[0, T] \times \Omega)$, and the functions $Z, Z-F$ are in $L^{2}\left((0, T) \times \Omega ; \mathbb{R}^{3}\right)$ by (iii) of Definition 4.12. Let $[a, b] \subset(0, T)$ be such that $\operatorname{supp}(\zeta) \subset[a, b]$. Then for sufficiently small $\varepsilon$, 
$\partial_{t} Z^{\varepsilon}=J(Z-F) * \eta_{\varepsilon}(t, x)$ in $(a, b) \times \mathbb{R}^{3}$, and $\left(Z^{\varepsilon}, \partial_{t} Z^{\varepsilon}\right) \rightarrow(Z, J(Z-F))$ in $L^{2}\left((a, b) \times \Omega ; \mathbb{R}^{3} \times \mathbb{R}^{3}\right)$ as $\varepsilon \rightarrow 0$. Then, integrating by parts to get

$$
\int_{0}^{T} \int_{\Omega} \zeta^{\prime}(t) \xi\left(Z_{t}^{\varepsilon}(x)\right) d x d t=-\int_{0}^{T} \int_{\Omega} \zeta(t) \nabla \xi\left(Z_{t}^{\varepsilon}(x)\right) \cdot \partial_{t} Z_{t}^{\varepsilon}(x) d x d t
$$

and using that $\xi, \nabla \xi, D^{2} \xi$ are bounded, we get that the left and right hand sides of the above equality converge to the left and right hand sides of (68), respectively. Indeed, denoting by $R_{\varepsilon}$ and $R$ the right-hand sides of (69) and (68) respectively, we have

$$
\begin{aligned}
&\left|R-R_{\varepsilon}\right| \leq \int_{a}^{b} \int_{\Omega}\left(\left\|D^{2} \xi\right\|_{L^{\infty}\left(\mathbb{R}^{3}\right)}\left|Z_{t}^{\varepsilon}(x)-Z_{t}(x)\right|\left|Z_{t}(x)-F_{t}(x)\right|\right. \\
&\left.+\|D \xi\|_{L^{\infty}\left(\mathbb{R}^{3}\right)}\left|\partial_{t} Z_{t}^{\varepsilon}(x)-J\left[Z_{t}(x)-F_{t}(x)\right]\right|\right) d x d t \\
& \leq C\left(\left\|Z_{t}(x)-F_{t}(x)\right\|_{L^{2}((0, T) \times \Omega)}\left\|Z_{t}^{\varepsilon}-Z_{t}\right\|_{L^{2}((a, b) \times \Omega)}\right. \\
&\left.+\left\|\partial_{t} Z_{t}^{\varepsilon}(x)-J\left[Z_{t}(x)-F_{t}(x)\right]\right\|_{L^{2}((a, b) \times \Omega)}\right) \rightarrow 0 .
\end{aligned}
$$

Convergence of the left-hand sides is proved similarly. This shows (68).

Next, (48) implies

$$
\left(\bar{\gamma}_{t} \circ \nabla P_{t} \circ \bar{\gamma}_{t}\right)(X)=\bar{\gamma}_{t}(X) \quad \text { for every } t \in(0, T) \text { and } \alpha(t, \cdot) \text {-a.e. } X \in \mathbb{R}^{3} .
$$

Using $\bar{\gamma}_{t \#} \alpha_{t}=\mu_{t}$, we get

$$
\bar{\gamma}_{t} \circ \nabla P_{t}(x)=x \text { for every } t \in(0, T) \text { and } \mu_{t} \text {-a.e. } x \in \Omega \text {. }
$$

In view of $F_{t} \# \chi=\mu_{t}$, we have

$$
\bar{\gamma}_{t} \circ Z_{t}(x)=\bar{\gamma}_{t} \circ \nabla P_{t} \circ F_{t}(x)=F_{t}(x) \text { for every } t \in(0, T) \text { and } \chi \text {-a.e. } x \in \Omega \text {. }
$$

Then we can rewrite (68) as

$$
\int_{0}^{T} \int_{\Omega} \zeta^{\prime}(t) \xi\left(Z_{t}(x)\right) d x d t=-\int_{0}^{T} \int_{\Omega} \zeta(t) \nabla \xi\left(Z_{t}(x)\right) \cdot J\left[Z_{t}(x)-\bar{\gamma}_{t} \circ Z_{t}(x)\right] d x d t,
$$

and using (66) to change variables in the right-hand side, we get

$$
\int_{0}^{T} \int_{\Omega} \zeta^{\prime}(t) \xi\left(Z_{t}(x)\right) d x d t=-\int_{0}^{T} \int_{\mathbb{R}^{3}} \zeta(t) \nabla \xi(X) \cdot J\left[X-\bar{\gamma}_{t}(X)\right] d \alpha_{t}(X) d t,
$$

Combining with (67), we get

$$
\int_{0}^{T} \int_{\mathbb{R}^{3}} \zeta^{\prime}(t) \xi(X) d \alpha_{t} d t=-\int_{0}^{T} \int_{\mathbb{R}^{3}} \zeta(t) \nabla \xi(X) \cdot J\left[X-\bar{\gamma}_{t}(X)\right] d \alpha_{t}(X) d t
$$

which is $(65)$ in the case $\varphi(t, X)=\zeta(t) \xi(X)$.

QED.

We finish with an observation concerning energy conservation along weak Lagrangian solutions of (1). 
Corollary 5.2. Let $(P, F)$ be a weak Lagrangian solution of (1) in the sense of Definition 4.12. Then

$$
\text { the function }[0, T) \ni t \rightarrow \int_{\Omega}\left|y-\nabla P_{t}(y)\right|^{2} d y \text { is constant. }
$$

Proof: Due to (59), we infer that the dual-space velocity $U(t, X)=X-\bar{\gamma}_{t}(X)$ satisfies

$$
\|U(t, \cdot)\|_{L^{2}\left(\alpha_{t} ; R^{3}\right)} \leq R_{0}\left[\mathcal{L}^{3}(\Omega)\right]^{1 / 2}+\left(\int_{\mathbb{R}^{3}}|X|^{2} d \alpha_{t}(X)\right)^{1 / 2} .
$$

But

$$
\int_{\mathbb{R}^{3}}|X|^{2} d \alpha_{t}(X)=\left\|\nabla P_{t}\right\|_{L^{2}\left(\Omega ; \mathbb{R}^{3}\right)}^{2} \text { for all } t \in[0, T),
$$

which, since $P \in C\left([0, \infty) ; H^{1}(\Omega)\right)$, implies the local boundedness in time of the $L^{2}\left(\alpha_{t} ; \mathbb{R}^{3}\right)$ norm of the velocity $U(t, \cdot)$, boundedness required by Theorem 5.2 in [4]. Furthermore, it follows that the path $t \rightarrow \alpha_{t}$ lies in $A C^{2}\left(0, T ; \mathcal{P}_{2}\left(\mathbb{R}^{3}\right)\right)$, see e.g. [4, page 24]. Thus, in light of Theorem 5.1 , we can apply Theorem 5.2 in [4] to conclude.

QED.

\section{Acknowledgements}

The authors would like to thank M. Cullen for his valuable suggestions and comments. The work of Mikhail Feldman was supported in part by the National Science Foundation under Grants DMS-0800245, DMS-1101260, and the Vilas Award by the University of Wisconsin-Madison. This work was partially supported by a grant from the Simons Foundation (\#246063 to Adrian Tudorascu).

\section{References}

[1] L. Ambrosio. Transport equation and Cauchy problem for BV vector fields. Invent. Math., 158 (2004), 227-260.

[2] L. Ambrosio, M. Colombo, G. De Philippis, A. Figalli. Existence of Eulerian solutions to the semigeostrophic equations in physical space: the 2-dimensional periodic case. Comm. Part. Diff. Eq. 37, No. 12 (2012), 2209-2227.

[3] L. Ambrosio, M. Colombo, G. De Philippis, A. Figalli "A global existence result for the semigeostrophic equations in three dimensional convex domains", Preprint arXiv:1205.5435, 2012 .

[4] L. Ambrosio, W. Gangbo. Hamiltonian ODE in the Wasserstein spaces of probability measures. Comm. Pure Appl. Math. 61 (2008), 18-53.

[5] L. Ambrosio, N. Gigli and G. Savaré. Gradient flows in metric spaces and the Wasserstein spaces of probability measures. Lectures in Mathematics, ETH Zurich, Birkhäuser, 2005.

[6] J.-D. Benamou, Y. Brenier. Weak existence for the Semi-Geostrophic equations formulated as a coupled Monge-Ampere/transport problem. SIAM J. Appl. Math., 58 (1998), no. 5, $1450-1461$. 
[7] Y. Brenier. Polar factorization and monotone rearrangement of vector-valued functions. Comm. Pure Appl. Math. 44 (1991), no 4, 375-417.

[8] M. Cullen. Private communication.

[9] M. Cullen and M. Feldman. Lagrangian solutions of Semi-Geostrophic equations in physical space. SIAM J. Math. Anal. 37 (2006), no 5, 1371-1395.

[10] M. Cullen and W. Gangbo. A variational approach for the 2-dimensional semi-geostrophic shallow water equations. Arch. Rat. Mech. Anal. 156 (2001), 241-273.

[11] M. Cullen, W. Gangbo, G. Pisante. The Semigeostrophic Equations discretized in reference and dual variables. Arch. Rational Mech. Anal. 185 (2007), 341-363.

[12] M. Cullen, H. Maroofi. The fully compressible semi-geostrophic system from meteorology. Arch. Ration. Mech. Anal. 167 (2003), no. 4, 309-336.

[13] G. De Philippis, A. Figalli. $W^{2,1}$ regularity for solutions of the Monge-Ampère equation. Invent. Math. (2012) DOI: 10.1007/s00222-012-0405-4.

[14] J.C. O Faria, M.C. Lopes Filho, H.J. Nussenzveig Lopes. Weak stability of Lagrangian solutions to the Semi-Geostrophic equations. Nonlinearity 22 (2009), 2521-2539.

[15] W. Gangbo, T. Nguyen and A. Tudorascu. Euler-Poisson systems as action-minimizing paths in the Wasserstein space. Arch. Rational Mech. Anal. 192, No. 3 (2009), 419-452.

[16] B. Hoskins. The Geostrophic Momentum Approximation and the Semi-Geostrophic Equations. J. Atmos. Sci. 32, No. 2 (1975), 233-242.

[17] G. Loeper. A fully nonlinear version of the incompressible Euler equations: the Semigeostrophic system. SIAM J. Math. Anal. 38, No. 3 (2006), 795-823.

[18] M.C. Lopes Filho, H.J. Nussenzveig Lopes Existence of a weak solution for the semigeostrophic equation with integrable initial data. Proc. Roy. Soc. Edinburgh Sect. A 132 (2002), no. 2, 329-339.

[19] A. Tudorascu. On the velocities of flows consisting of cyclically monotone maps. Indiana Univ. Math. J., 59, No. 3 (2010), pp. 929-955.

[20] C. Villani. Topics in optimal transportation. Graduate Studies in Mathematics 58, American Mathematical Society, 2003. 